\title{
25. INTERSTITIAL WATER CHEMISTRY AND DIAGENESIS OF BIOGENIC SEDIMENTS FROM THE EASTERN EQUATORIAL PACIFIC, DEEP SEA DRILLING PROJECT LEG $85^{1}$
}

\author{
Paul M. Stout, Scripps Institution of Oceanography
}

\begin{abstract}
The concentration changes in pore waters of dissolved calcium, magnesium, sulfate, strontium, and silica and of alkalinity are controlled by diagenetic reactions occurring within the biogenic sediments of DSDP Sites 572, 573, and 574 . Downcore increases in dissolved $\mathrm{Sr}^{2+}$ indicate recrystallization of calcite, and increases in dissolved $\mathrm{SiO}_{2}$ reflect dissolution of amorphous silica. Minor gradients in dissolved $\mathrm{Ca}^{2+}$ and $\mathrm{Mg}^{2+}$ suggest little if any influence from reactions involving volcanic sediments or basalt. Differences in interstitial water profiles showing the downhole trends of these chemical species mark variations in carbonate and silica diagenesis, sediment compositions, and sedimentation rate histories among the sites.

The location and extent of carbonate diagenesis in these sediments are determined from $\mathrm{Sr} / \mathrm{Ca}$ distributions between the interstitial waters and the bulk carbonate samples. Pore water strontium increases in the upper 100 to $250 \mathrm{~m}$ of sediment are assumed to reflect diffusion from underlying zones where calcite recrystallization has occurred. On the basis of calculations of dissolved strontium production and comparisons between observed and calculated "equilibrium" $\mathrm{Sr} /$ Ca ratios of the solids, approximately 30 to $50 \%$ of the carbonate has recrystallized in these deeper intervals. These estimates agree with the observed amounts of chalk at these sites. Variations in $\mathrm{Sr} / \mathrm{Ca}$ ratios of these carbonates reflect differences in calcareous microfossil content, in diagenetic history, and, possibly, in changes in seawater $\mathrm{Sr} / \mathrm{Ca}$ with time.

Samples of porcelanite recovered below $300 \mathrm{~m}$ at Site 572 suggest formation at temperatures 20 to $30^{\circ} \mathrm{C}$ greater than ones estimated assuming oceanic geothermal gradients from sedimentary sections similar to those recovered on Leg 85 . The higher temperatures may partially account for higher $\mathrm{Sr} / \mathrm{Ca}$ ratios determined for recrystallized carbonates from this site.
\end{abstract}

\section{INTRODUCTION}

Recovery of complete and undisturbed sedimentary sections by the Glomar Challenger has enabled detailed investigations of the physical and chemical changes that transform unconsolidated marine sediments into lithified rocks. These transformations include the progression from calcareous ooze to chalk and limestone, and from siliceous ooze to porcelanite and chert.

Several previous studies on diagenesis of calcareous deposits from DSDP sites in the Pacific Ocean concentrated on textural evidence to examine downhole recrystallization of carbonates (e.g., Schlanger and Douglas, 1974; Matter et al., 1975; van der Lingen and Packham, 1975). Recently, Baker et al. (1982) and Elderfield et al. (1982) used a geochemical approach to examine carbonate diagenesis from the same regions. Concentration distributions of calcium, magnesium, strontium, and variations in ${ }^{87} \mathrm{Sr} /{ }^{86} \mathrm{Sr}$ and $\delta^{18} \mathrm{O}$ between the solid phases and interstitial waters were compared to assess the amount of diagenetic alteration. Baker et al. (1982) demonstrated that significant calcite recrystallization occurs at subbottom depths less than $200 \mathrm{~m}$. They showed how $\mathrm{Sr} / \mathrm{Ca}$ ratios in sediments progressively change downhole, in accordance with predicted equilibrium distributions, in response to calcite recrystallization. Elderfield et al. (1982) further substantiated these conclusions with stron-

\footnotetext{
${ }^{1}$ Mayer, L., Theyer, F., et al., Init. Repts. DSDP, 85: Washington (U.S. Govt. Printing Office).

2 Address: Scripps Institution of Oceanography, University of California, San Diego, La Jolla, CA 92093.
}

tium isotope data. Identical values of ${ }^{87} \mathrm{Sr} /{ }^{86} \mathrm{Sr}$ ratios were determined for the carbonates and interstitial waters at the same depths where $\mathrm{Sr} / \mathrm{Ca}$ ratios agreed with predicted equilibrium distributions.

Leg 85 afforded an opportunity to expand our understanding of diagenetic changes within calcareous deposits (Fig. 1). Use of the variable length hydraulic piston corer, combined with complete coring of sections drilled to basement and closely spaced interstitial water sampling, enabled more detailed examination of earlier stages of diagenesis than was possible with rotary drilling methods. The overall objective of the leg, which was to conduct a detailed investigation of the paleoceanographic and paleoclimatic history of the region, allowed us to consider the influences that varying oceanographic and tectonic conditions have on diagenetic processes (and have had in the past). The effects of diagenetic processes on paleoceanographic reconstructions could also be examined.

The sedimentary successions cored during this leg are almost exclusively biogenic, composed predominantly of calcareous nannofossils and varying mixtures of foraminifers, radiolarians, and diatoms (Fig. 2). The sediments accumulated nearly continuously, with only short intervals of nonaccumulation or erosion (Fig. 3). This chapter presents data on the chemistry of interstitial waters and the $\mathrm{Sr} / \mathrm{Ca}$ distributions between the carbonate sediments and interstitial waters. These data are used to interpret and identify the extent of diagenetic alteration within the sediments and to determine the important factors that control the observed distributions and trends. Results and discussions are for Sites 572, 573, and 574, the three sites drilled to basement (Figs. 2 and 3). 


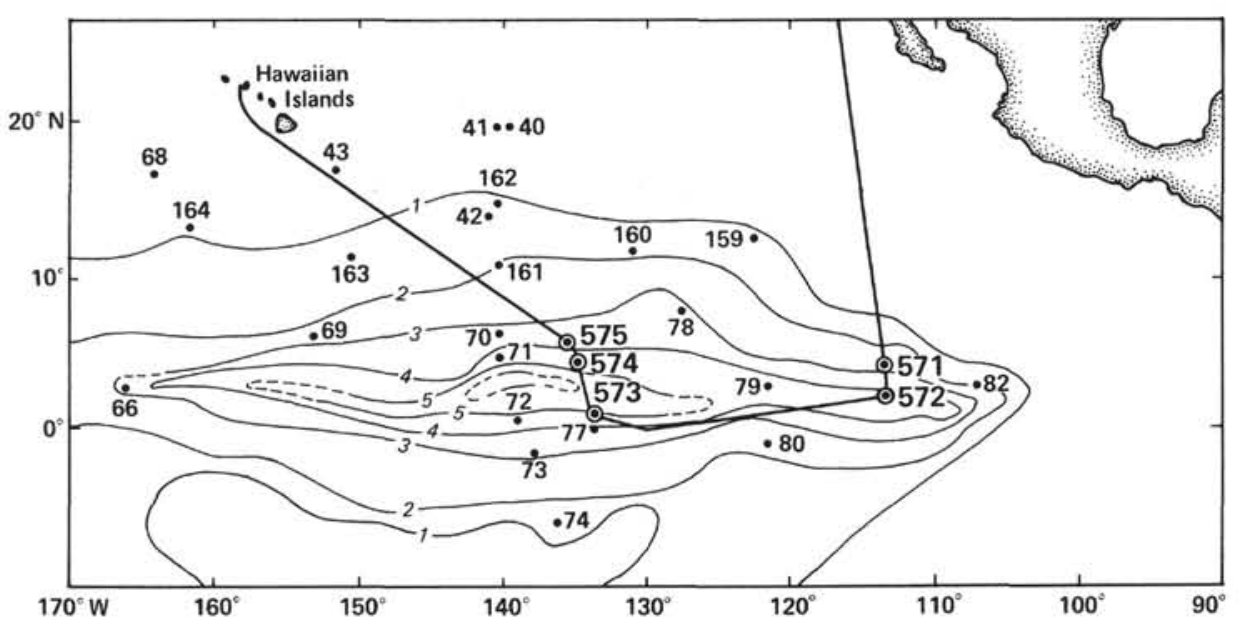

Figure 1. Cruise track and site locations of Leg 85 in relationship to acoustic sediment thicknesses (contours in tenths of seconds of two-way travel-time) and previous DSDP operations (Legs 5, 8, 9, and 16). $=$ Leg $85 ; 0=$ other DSDP legs.

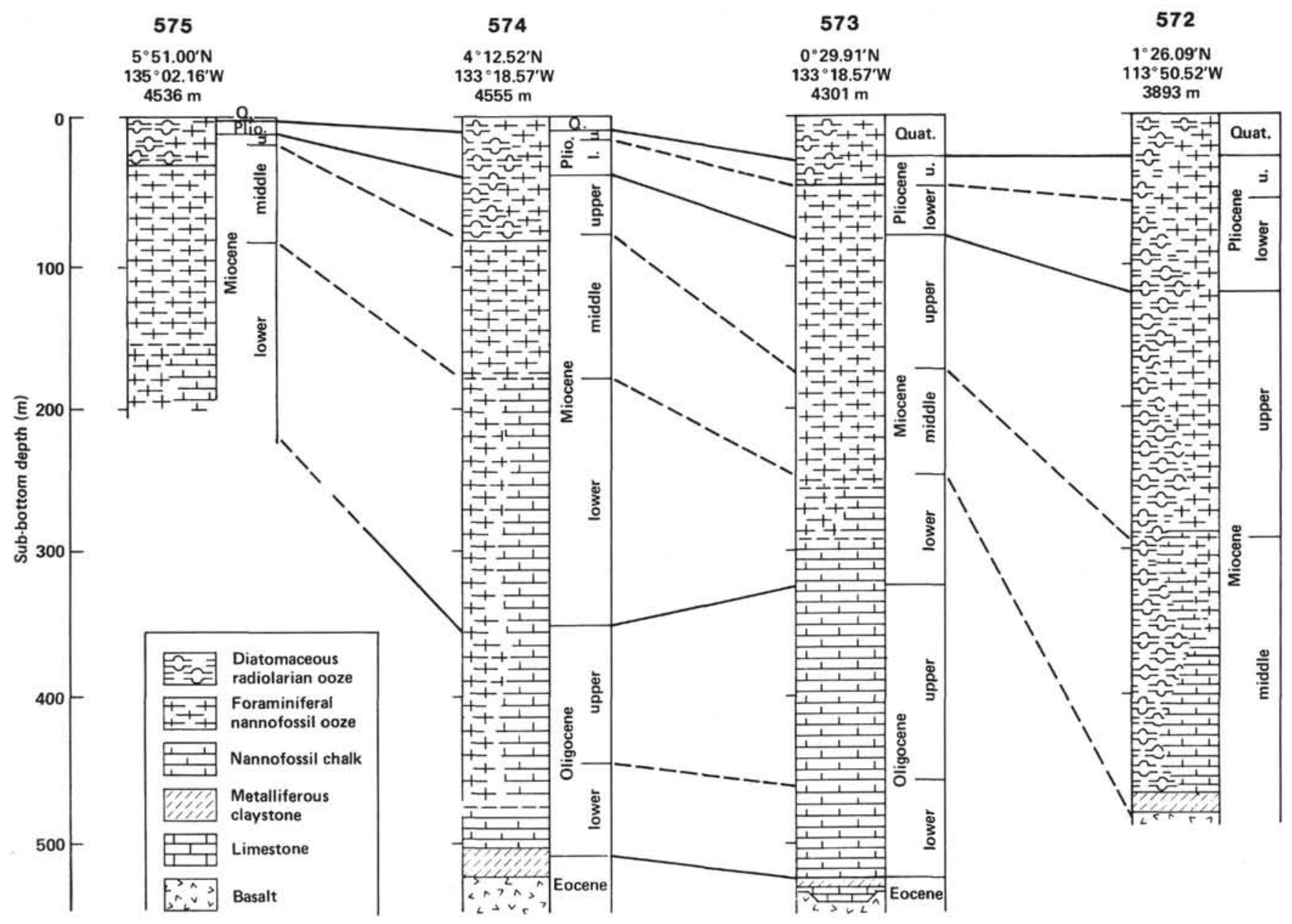

Figure 2. Lithologic columns and stratigraphy of Sites 572, 573, 574, and 575 . 


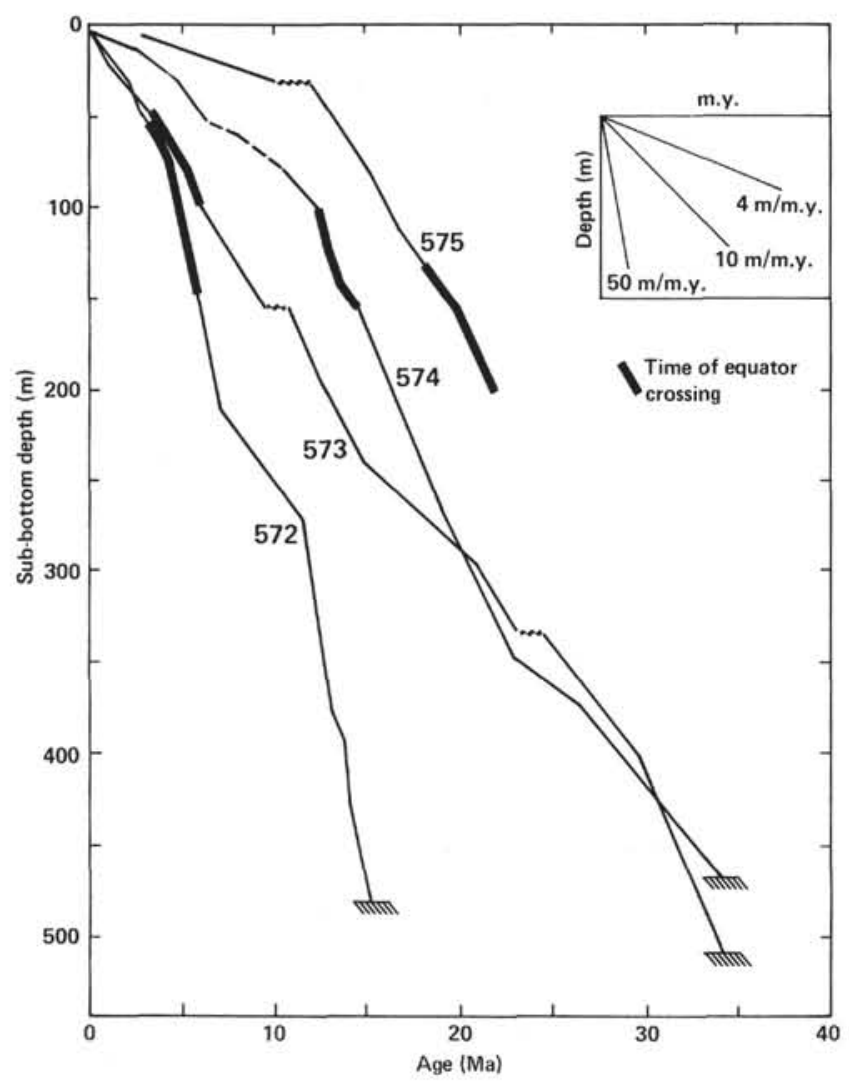

Figure 3. Sediment accumulation rates at Sites 572, 573, 574, and 575, as determined on board ship.

The time scale used is as discussed in the Introduction (this volume); sedimentation rate curves are as determined on board ship.

\section{METHODS}

Interstitial water samples were obtained at intervals between 1 and $30 \mathrm{~m}$ for all sites. Slightly larger intervals $(50 \mathrm{~m})$ were required in a few places because of concern about sampling major stratigraphic boundaries. Shipboard processing of samples and analytical techniques for the various ions are reported elsewhere (Gieskes, 1973, 1974; Gieskes et al., 1982). All squeezing of samples on board was performed at room temperature. We usually recovered about $50 \mathrm{ml}$ of fluid, except in a few cases where induration was extreme and/or drilling disturbance was severe, but at least $15 \mathrm{ml}$ were always recovered.

Concentrations of calcium and strontium in carbonate samples were determined following leaching of powdered samples by an acetic acid-sodium acetate solution ( $\mathrm{pH}$ 5). Calcium was determined by EGTA titration and strontium by flame atomic absorption. No corrections were made for salt content resulting from drying $\left(60^{\circ} \mathrm{C}\right)$. Experience has shown that for squeezed samples, corrections for strontium and calcium are insignificant (J. Gieskes, pers. comm., 1983) The influence of ion exchange reactions for strontium concentrations, which has not been adequately studied (Gieskes, 1978), is assumed to be small due to negligible amounts of clay minerals.

Insoluble residues of the carbonates were quantitatively determined, and the samples were analyzed by X-ray diffraction (XRD). Selected samples of the acid-leached sediment and original sediments and rocks were examined by petrographic and scanning electron microscopy (SEM) having an attached X-ray energy dispersive unit. A thorough examination of sediment texture and composition has not been completed. Data on porosities, weight percentage calcium carbonate, electrical conductivity (used to determined sediment diffusion coefficients), and microfossil abundances are from shipboard results reported elsewhere in this volume.

\section{INTERSTITIAL WATER CHEMISTRY}

Concentration versus depth profiles for Sites 572, 573, and 574 are plotted in Figures 4, 5, and 6. Table 1 contains sample location data and the concentrations determined for all species analyzed. Profiles for dissolved calcium, strontium, magnesium, silica, and sulfate and for alkalinity are presented. In general, the profiles from all sites show similar trends and similar changes in magnitude going downhole from overlying seawater. Maximum and minimum values, however, do not occur at the same depths. The slight contrasts in concentration changes and the different locations of maxima and minima reflect differences in sediment composition and in the loci of intervals undergoing enhanced diagenetic alterations.

The strontium profiles reveal steady concentration increases until near the maximum value, where an interval of roughly constant concentration occurs. Below these intervals, the values steadily decrease as basement is approached. I use these similar trends in dissolved strontium to divide each sedimentary section into three zones. Zone I corresponds to the interval where the concentrations steadily increase, Zone II to the intervals where the concentrations are approximately constant at maxima, and Zone III to the lowest sections, where concentrations steadily decrease. The depth relationships for each zone are summarized in Table 2 . The sediment sections are divided in this manner for purposes of interpreting the carbonate recrystallization histories of these sites (discussed in detail below).

The observed increases in dissolved strontium are attributed to diagenetic recrystallization of calcite. Calcareous microfossils incorporate more strontium into their skeletons than predicted from equilibrium distribution coefficients for inorganic calcite (Katz et al., 1972; Baker et al., 1982). During recrystallization of calcite, the calcite dissolved and reprecipitated with lesser amounts of strontium, generating the observed strontium increase in pore waters (e.g., Sayles and Manheim, 1975; Gieskes, 1981). Other possible sources for strontium increases in solution include alteration of volcanic matter in the sediments or alteration of the underlying basaltic layer (Elderfield et al., 1982; Elderfield and Gieskes, 1982). However, such sources are not present here; the amount of volcanic sediment is negligible, and the gradients in dissolved calcium and magnesium are very slight. In deepsea sections where extensive alteration of oceanic layer II is important, fivefold or greater increases in calcium and decreases in magnesium are observed (Lawrence et al., 1975; Gieskes, 1981; Gieskes and Lawrence, 1981).

The trends in alkalinity resemble those for strontium at each site. Sulfate profiles, in contrast, display opposite trends. Increases in alkalinity probably represent production from bacterial sulfate reduction. Sulfate decreases and alkalinity increases are largest at Site 572 , for which sediment accumulation rates are highest (Figs. 3 to 4). The possible relationships between sulfate, alkalinity, and strontium profiles and sedimentation rate histories cannot be explained definitively at present. However, Sayles and Manheim (1975) and Gieskes and Johnson (1985) suggested that higher sedimentation rates cause 


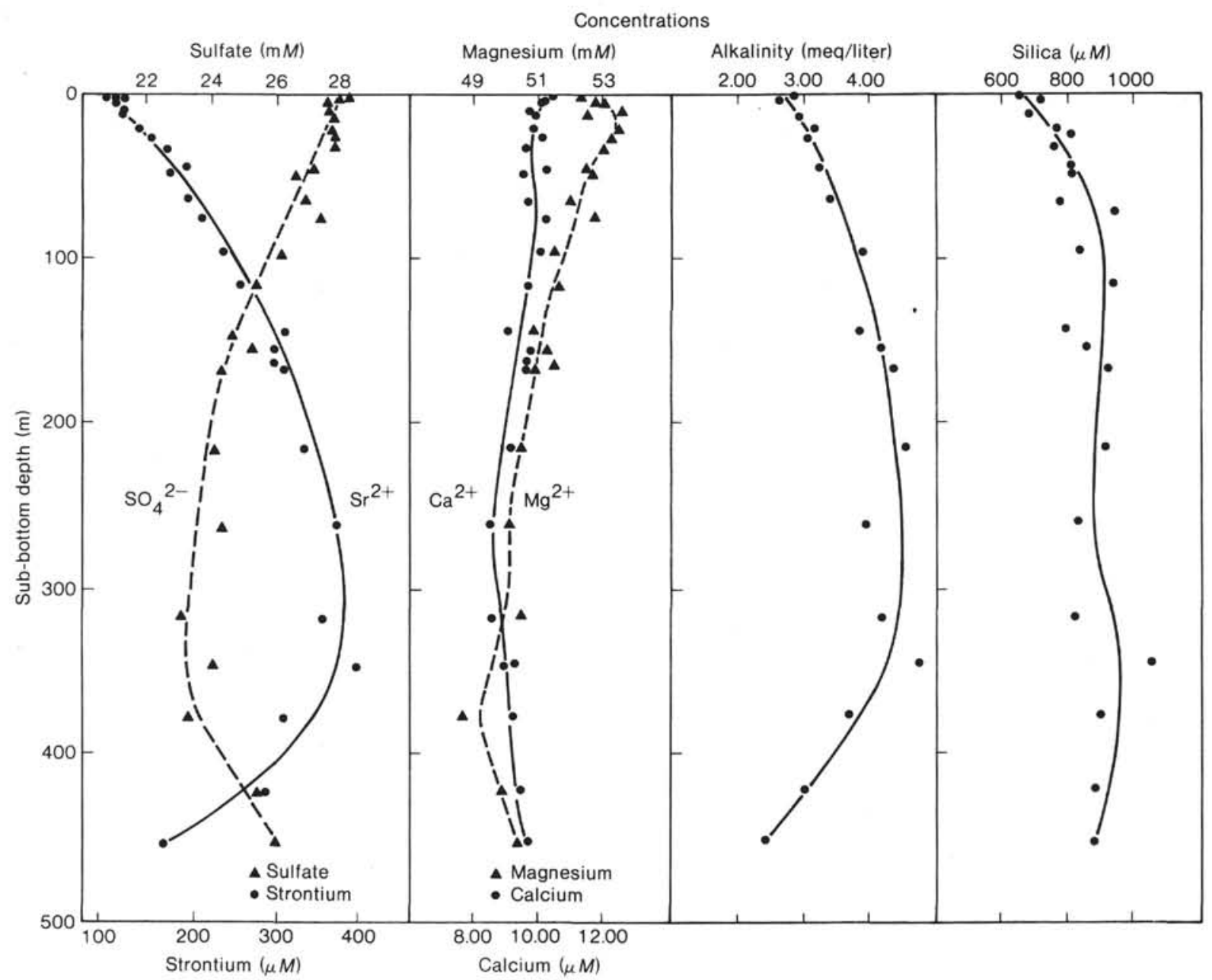

Figure 4. Interstitial water chemistry for Site 572.

burial of more organic matter, which in turn leads to more sulfate reduction and possibly higher rates of carbonate recrystallization. The increase in carbonate recrystallization is manifest by increases in dissolved strontium. The connection between sedimentation rate and carbonate diagenesis is discussed more fully in following sections of this chapter.

The dissolved silica profiles for the three sites are similar. The values increase from near-surface lows of about $600 \mu M$ to concentrations between 800 and $1000 \mu M$. Some of the scatter in silica concentrations over short depth ranges is caused by differences in squeezing temperatures and by the amount of siliceous components in the sediments. In some samples taken at identical depths, and which contain similar percentages of noncarbonate components, the dissolved silica concentrations were 15 to $25 \%$ higher for sediments having higher temperatures $\left(10\right.$ to $\left.15^{\circ} \mathrm{C}\right)$ at time of squeezing. There is agreement between the average dissolved silica concentrations and the average percentages of noncarbonate in the sediments from which the pore waters were recovered. I assume that most noncarbonate material is biogenic silica; this is supported by petrographic observations and XRD results that indicate only trace amounts of nonbiogenic components. Site 572 had the highest values: $836 \mu M$ dissolved silica and $27 \%$ noncarbonate. Site 572 was intermediate with $776 \mu M$ silica and $20 \%$ noncarbonate; Site 573 had the lowest with $723 \mu M$ silica and $16 \%$ noncarbonate. Site 572 , the easternmost site, also had the highest individual dissolved silica concentration of all samples: a value of $1055 \mu M \mathrm{SiO}_{2}$ was found for a sample from $345 \mathrm{~m}$ sub-bottom. Small pieces (several centimeters in diameter) of laminated porcelanite were recovered below $300 \mathrm{~m}$ at irregular intervals at this site. At Site 574, silica concentrations between 380 and $480 \mathrm{~m}$ are close to the maximum values of Site 572 , yet no porcelanite was recovered. This may reflect nonrecovery of samples that may have been present at Site 574. Alternatively, the absence of porcelanite may result from a higher geothermal gradient at Site $\mathbf{5 7 2}$.

\section{Sr/Ca DISTRIBUTIONS OF CARBONATES AND INTERSTITIAL WATERS}

The strontium and calcium distributions between the pore fluids and sediments are compared to determine the extent of carbonate diagenesis in these sediments. Following the approach of Baker et al. (1982), "equilibrium" values for the $\mathrm{Sr} / \mathrm{Ca}$ ratios are calculated and 


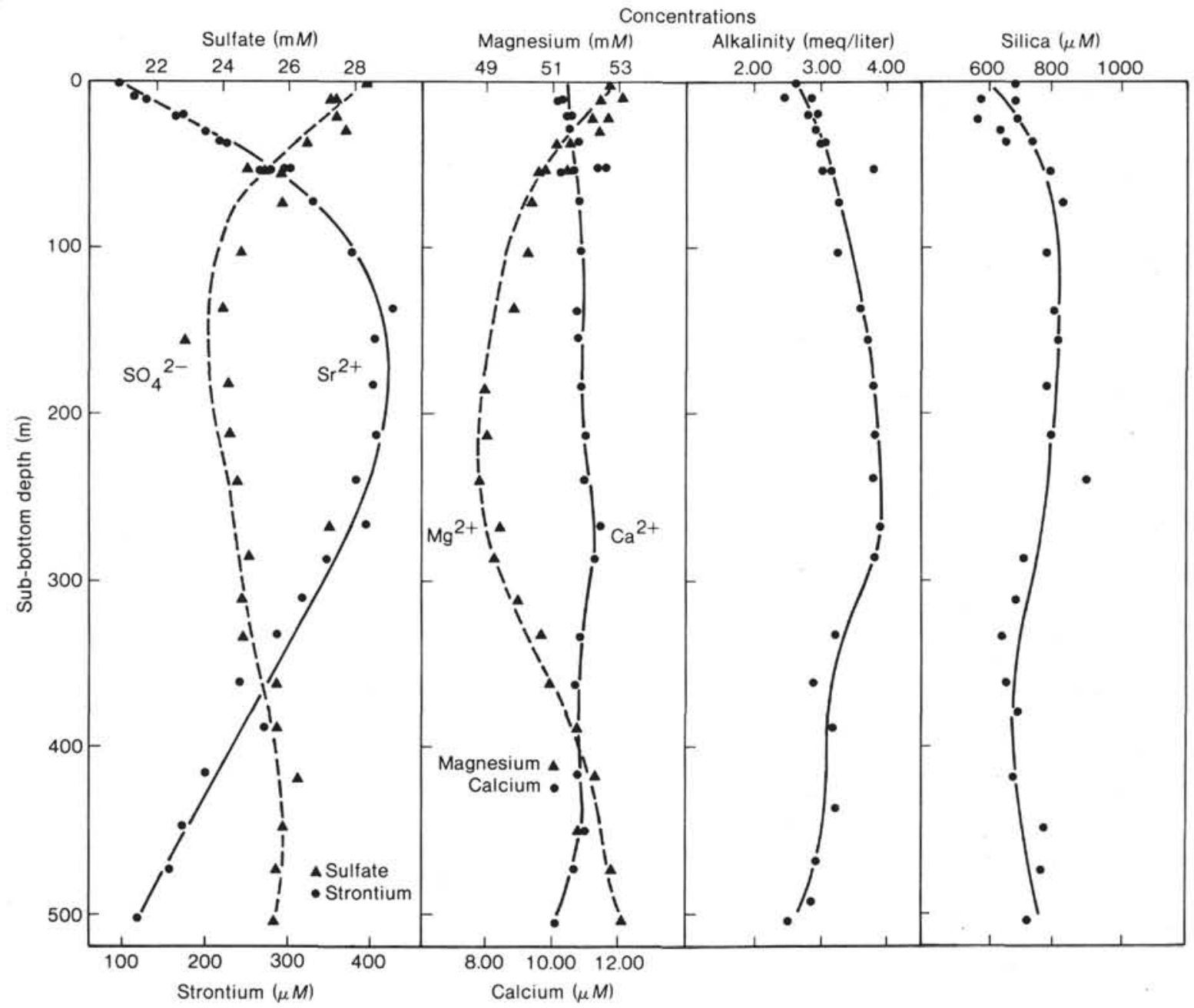

Figure 5. Interstitial water chemistry for Site 573.

plotted with the ratios determined for the leached bulk samples. The "equilibrium" values are calculated from the equation

$$
\mathrm{Sr} / \mathrm{Ca}=k_{\mathrm{Sr}}\left(\mathrm{Sr}^{2+} / \mathrm{Ca}^{2+}\right)
$$

where

$$
\begin{array}{ll}
\mathrm{Sr} / \mathrm{Ca} & =\text { molar ratio in carbonates } \\
k_{\mathrm{Sr}} & =\begin{array}{l}
\text { experimentally determined distribution } \\
\text { coefficient for strontium in low- } \mathrm{Mg}
\end{array} \\
& \text { calcite } \\
\mathrm{Sr}^{2+} / \mathrm{Ca}^{2+}= & \text { molar ratio in interstitial waters }
\end{array}
$$

The distribution coefficient $k_{\mathrm{Sr}}$ was corrected for temperature and solution composition after Baker et al. (1982) assuming a $40^{\circ} \mathrm{C} / \mathrm{km}$ geothermal gradient (Sites 71 and 72, Von Herzen et al., 1971).

There is continued disagreement on the appropriate value of $k_{\mathrm{Sr}}$ for inorganic calcite. Recent results by Mucci and Morse (1983), extrapolated to low-Mg calcite, support early values reported by Kinsman (1969) and Holland et al. (1964), which are an order of magnitude higher than those determined by Katz et al. (1972) and
Baker et al. (1982). The experiments conducted by the latter groups were performed at very slight calcite supersaturation values, a situation that more closely resembles diagenetic conditions occurring in deep-sea carbonate sediments. Furthermore, if the larger distribution coefficient were appropriate in these sediments, one would not expect to see strontium increases in pore waters, nor would one observe the decreases in the $\mathrm{Sr} / \mathrm{Ca}$ ratios in the solids, which has been clearly documented (Baker et al., 1982; Elderfield et al., 1982).

The calculated $\mathrm{Sr} / \mathrm{Ca}$ equilibrium ratios and the observed ratios in bulk carbonate values are plotted in Figures 7, 8, and 9 and included in Table 3 . The curves for the $\mathrm{Sr} / \mathrm{Ca}$ equilibrium values closely resemble the dissolved strontium profiles (Figs. 4, 5, 6), reflecting the minor changes with depth in interstitial calcium concentrations. Overall, the dissolved calcium decreases slightly with depth at Site 572 but increases with depth at the other two sites. The slight changes in dissolved calcium affect the maximum $\mathrm{Sr} / \mathrm{Ca}$ equilibrium values estimated for each site. For example, the maximum equilibrium value is $2.02 \times 10^{-3}$ at Site 572 , compared to $1.48 \times$ $10^{-3}$ for Site 574 . In contrast, the maximum observed dissolved strontium concentrations at these two sites are 


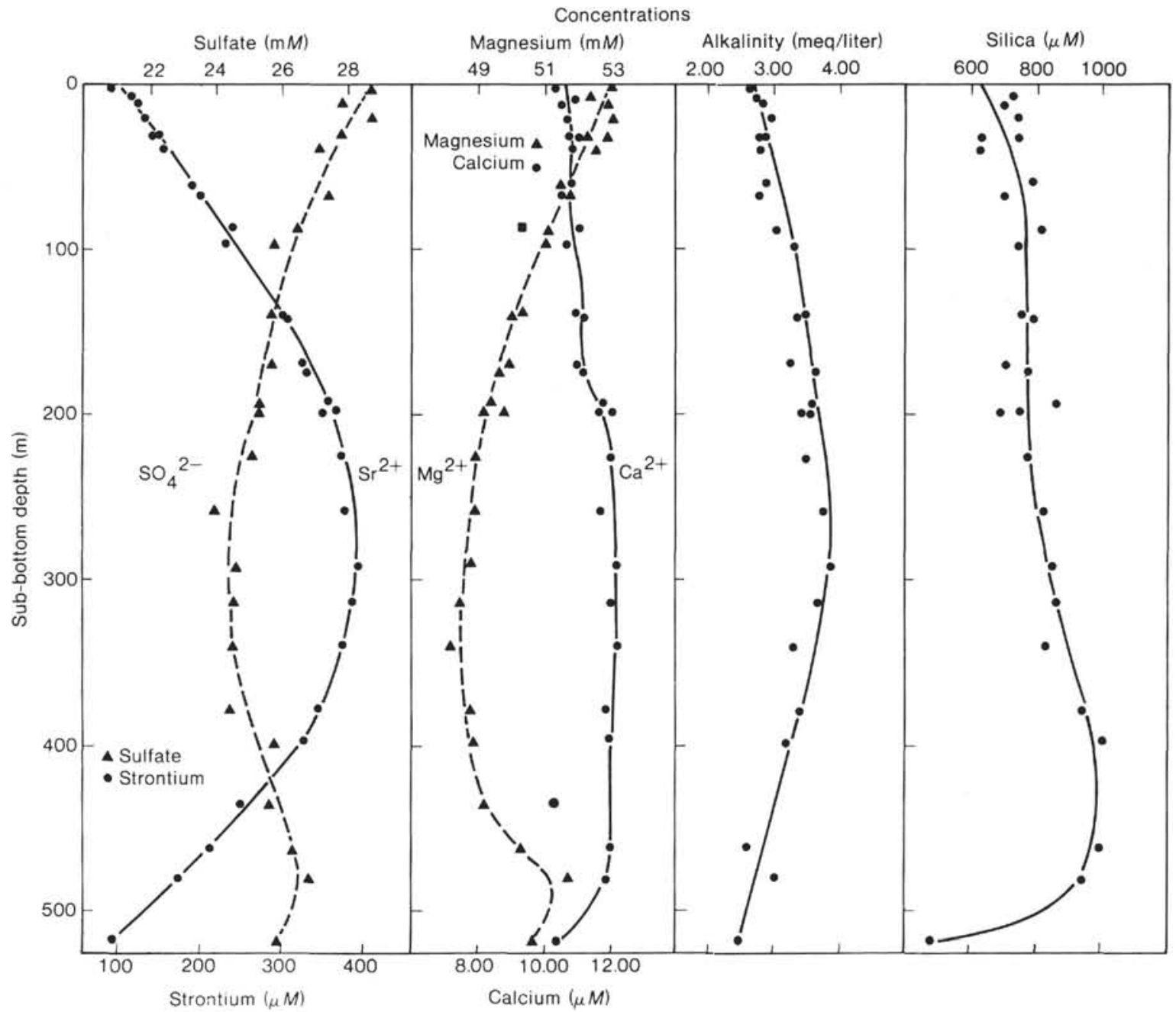

Figure 6. Interstitial water chemistry for Site 574 .

virtually identical: $397 \mu M \mathrm{Sr}^{2+}$ for Site 572 and $391 \mu M$ $\mathrm{Sr}^{2+}$ for Site 574 . The reasons for the slight variations in calcium are unknown. Whatever the reasons, the interstitial calcium variations strongly influence the predicted $\mathrm{Sr} / \mathrm{Ca}$ ratios and thus the interpretation of calcite recrystallization at each site.

There is little agreement between the observed $\mathrm{Sr} / \mathrm{Ca}$ ratios and the calculated equilibrium values for the three sites (Figs. 7 to 9). Except for a few isolated points, the measured values are significantly higher than the predicted values. The observed values more closely approach the equilibrium ratios in the 100 to 150 -m-thick zones near the predicted $\mathrm{Sr} / \mathrm{Ca}$ maxima where the dissolved $\mathrm{Sr}^{2+}$ concentrations reach approximately constant values (compare Figs. 4 to 6 with 7 to 9 ). These zones correspond to Zone II of Table 2 . The closest agreement between the observed and predicted equilibrium values in this zone implies that carbonate recrystallization is occurring most actively over these depth intervals. In Zone III, the larger disparity between predicted and observed ratios suggests that carbonate recrystallization is presently less active and that the $\mathrm{Sr} / \mathrm{Ca}$ values in the carbonates reflect older diagenetic conditions. In sediments within the uppermost zone, the differences between predicted and observed ratios suggest that carbonate recrystallization has not been significant.

$\mathrm{The} \mathrm{Sr} / \mathrm{Ca}$ ratios measured from the bulk carbonate leaches vary over a wide range throughout the three sites. The variations may be attributed to numerous factors, the more important ones including (1) differences in calcareous microfossil compositions of the sediments (variations in the nannofossil/foraminifer ratio); (2) chemical paleoceanographic effects, such as variations in the $\mathrm{Sr} / \mathrm{Ca}$ ratio or in the temperature of seawater with age, and consequently the $\mathrm{Sr} / \mathrm{Ca}$ ratios incorporated during growth of skeletal calcite; and (3) the amount of recrystallized calcite and the specific physicochemical conditions under which recrystallization occurred.

Concerning the first factor, chemical analyses of individual species of foraminifers reveal $\mathrm{Sr} / \mathrm{Ca}$ ratios in calcite ranging from $1.2 \times 10^{-3}$ to $1.7 \times 10^{-3}$ (Bender et al., 1975; Lorens et al., 1977; Elderfield et al., 1982). The bulk carbonate sediment samples are consistently higher in measured $\mathrm{Sr} / \mathrm{Ca}$ ratios than individual species of foraminifers of the same age. The bulk samples invariably contain nannofossils, implying that nannofos- 
Table 1. Interstitial water data, Leg 85 .

\begin{tabular}{|c|c|c|c|c|c|c|c|c|c|c|}
\hline $\begin{array}{c}\text { Sample } \\
\text { (interval in } \mathrm{cm} \text { ) }\end{array}$ & $\begin{array}{l}\text { Sub-bottom } \\
\text { depth (m) }\end{array}$ & $\mathrm{pH}$ & $\begin{array}{l}\text { Alkalinity } \\
\text { (meq/liter) }\end{array}$ & $\begin{array}{l}\mathrm{SO}_{4}^{2-} \\
(\mathrm{m} M)\end{array}$ & $\underset{(\mu M)}{\mathrm{SiO}_{2}}$ & $\begin{array}{l}\mathrm{Ca}^{2+} \\
(\mathrm{m} M)\end{array}$ & $\begin{array}{l}\mathrm{Mg}^{2+} \\
(\mathrm{mM} M)\end{array}$ & $\begin{array}{l}\mathrm{Sr}^{2+} \\
(\mu M)\end{array}$ & $\begin{array}{c}\mathrm{Cl} \\
\left(\% \%_{0}\right)\end{array}$ & $(\%)$ \\
\hline \multicolumn{11}{|l|}{ Hole 572} \\
\hline $1-1,115-135$ & 1 & 7.49 & 2.87 & 28.2 & - & 10.49 & 52.25 & 96 & 19.14 & \\
\hline $1-2,100-120$ & 2.5 & 7.58 & 2.64 & 27.7 & - & 10.19 & 52.67 & 101 & 19.34 & \\
\hline $1-3,100-120$ & 4 & 7.48 & 2.65 & 27.7 & - & 10.18 & 52.76 & 116 & 19.34 & \\
\hline \multicolumn{11}{|l|}{ Hole $572 \mathrm{~A}$} \\
\hline $1-1,0-20$ & 0.5 & 7.58 & 2.63 & 27.9 & 653 & 10.39 & 52.81 & 96 & 19.14 & \\
\hline $1-4,50-70$ & 5 & 7.60 & 2.38 & 27.5 & 716 & 10.08 & 53.03 & 101 & 19.12 & \\
\hline $2-1,0-20$ & 9.5 & 7.56 & 2.25 & 27.5 & - & 9.70 & 53.48 & 116 & 19.24 & \\
\hline $3-2,115-135$ & 22 & 7.51 & 3.16 & 27.6 & 765 & 9.84 & 53.46 & 131 & 19.36 & \\
\hline $4-4,95-115$ & 34 & 7.57 & 2.49 & 27.7 & 757 & 9.63 & 53.02 & 161 & 19.60 & \\
\hline $6-3,85-105$ & 50 & 7.46 & 2.60 & 26.5 & 816 & 9.47 & 52.64 & 167 & 19.51 & \\
\hline $9-3,85-105$ & 76 & 7.46 & 3.25 & 27.3 & 941 & 10.12 & 52.71 & 208 & 19.51 & \\
\hline $13-5,85-105$ & 117 & 7.40 & 3.47 & 25.4 & 939 & 9.64 & 51.61 & 255 & 19.31 & \\
\hline
\end{tabular}

Hole 572B

$\begin{array}{llllllllllll}2-3,123-143 & 164 & 7.50 & 3.99 & - & - & 9.66 & 51.50 & 296 & 19.27 & 35.2\end{array}$

Hole 572C

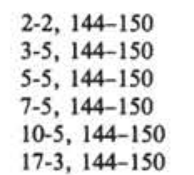

$\begin{array}{rr}13 & 7.38 \\ 27 & 7.39 \\ 46 & 7.39 \\ 66 & 7.41 \\ 97 & 7.43 \\ 145 & 7.40\end{array}$

$\begin{array}{ll}2.93 & 27.6 \\ 3.09 & 27.7 \\ 3.23 & 27.0 \\ 3.39 & 26.8 \\ 3.92 & 26.1 \\ 3.87 & 24.6\end{array}$

Hole 572D

$1-3,144-150$
$2-5,144-150$
$7-5,144-150$
$12-4,144-150$
$18-4,144-150$
$21-4,144-150$
$24-5,144-150$
$29-4,140-150$
$32-4,140-150$

155
168
215
261
318
346
377
423
454

$\begin{array}{ll}7.39 & 4.20 \\ 7.34 & 4.37 \\ 7.35 & 4.58 \\ 7.43 & 3.93 \\ 7.43 & 4.22 \\ 7.43 & 4.76 \\ 7.38 & 3.70 \\ 7.53 & 3.02 \\ 7.54 & 2.43\end{array}$

Hole 573

$1-1,144-150$
$2-6,144-150$
$3-6,144-150$
$5-5,144-150$
In situ I
In situ II
$7-5,140-150$
$9-5,140-150$
$12-5,140-150$
$16-5,140-150$

1.5
11
20.5
38
53
53
55
74
103
138

$\begin{array}{cc}7.39 & 2.61 \\ 7.29 & 2.84 \\ 7.33 & 2.97 \\ 7.31 & 3.05 \\ 7.66 & 3.76 \\ \overline{7.30} & -\overline{18} \\ 7.24 & 3.29 \\ 7.35 & 3.24 \\ 7.25 & 3.60\end{array}$

25.2
24.2
24.0
24.2
23.1
24.0
23.3
25.4
26.0

$\begin{array}{lrllll}685 & 9.89 & 52.44 & 113 & 19.10 & - \\ 810 & 10.08 & 53.26 & 142 & 19.31 & 35.5 \\ 816 & 9.47 & 52.64 & 185 & 19.39 & 35.5 \\ 778 & 9.65 & 52.04 & 190 & 19.49 & 35.5 \\ 833 & 10.03 & 51.46 & 231 & 19.19 & - \\ 795 & 9.04 & 50.93 & 309 & 18.92 & -\end{array}$

Hole 573A

$1-4,144-150$
$2-4,144-150$
$3-5,143-150$
$4-4,143-150$
$6-4,143-150$

10.5
21
30
37
55

7.38
7.33
7.28
7.30
7.33

2.48
2.83
2.93
3.00
3.01

28.4
27.3
-
-
$\overline{2}$
24.8
25.8
25.8
24.5
24.0

679
678
691
732
819
675
781
821
778
797

$\begin{array}{ll}10.42 & 52.68 \\ 10.22 & 53.37 \\ 10.60 & 52.15 \\ 10.59 & 52.68 \\ 11.40 & 51.52 \\ 11.51 & 50.77 \\ 10.70 & 50.77 \\ 10.81 & 50.38 \\ 10.83 & 50.25 \\ 10.79 & 49.82\end{array}$

$\begin{array}{rlllll}856 & 9.74 & 51.31 & 296 & 19.24 & 35.2 \\ 922 & 9.61 & 50.96 & 308 & 19.25 & 35.2 \\ 916 & 9.16 & 50.51 & 331 & 19.26 & 35.1 \\ 835 & 8.53 & 50.09 & 372 & 19.26 & 34.9 \\ 822 & 8.64 & 50.48 & 356 & 19.17 & 34.9 \\ 1055 & 9.14 & 49.89 & 397 & 19.32 & 34.9 \\ 906 & 9.20 & 48.67 & 308 & 19.14 & 34.6 \\ 887 & 9.43 & 49.94 & 285 & 19.29 & 35.1 \\ 887 & 9.69 & 50.32 & 161 & 19.34 & 35.2\end{array}$

Hole 573B

$2-5,143-150$
$5-5,144-150$
$8-5,140-150$
$11-4,140-150$
$14-4,140-150$
$16-4,140-150$
$19-2,65-95$
$21-3,140-150$
$24-3,140-150$
$27-2,140-150$
$30-2,140-150$
$33-5,140-150$

$\begin{array}{ll}156 & 7 \\ 184 & 7 \\ 212 & 7 \\ 239 & 7 \\ 268 & 7 \\ 287 & 7.31 \\ 312 & \\ 333 & 7 \\ 361 & 7.37 \\ 388 & 7.4 \\ 417 & 7.34 \\ 450 & 7.32\end{array}$

$\begin{array}{ccc}7.31 & 3.70 & 22.9 \\ 7.30 & 3.78 & 24.1 \\ 7.22 & 3.85 & 24.3 \\ 7.24 & 3.80 & 24.4 \\ 7.31 & 3.90 & 27.2 \\ 7.31 & 3.78 & 24.8 \\ - & - & 24.5 \\ 7.37 & 3.22 & 24.5 \\ 7.41 & 2.89 & 25.6 \\ 7.34 & 3.17 & 25.6 \\ 7.31 & 3.21 & 26.2 \\ 7.32 & 2.95 & 25.8\end{array}$

$\begin{array}{lcc}802 & 10.83 & - \\ 779 & 10.83 & 48.93 \\ 790 & 10.99 & 49.00 \\ 895 & 10.96 & 48.78 \\ - & 11.42 & 49.41 \\ 707 & 11.26 & 49.24 \\ 687 & - & 49.83 \\ 647 & 10.82 & 50.62 \\ 658 & 10.64 & 50.90 \\ 690 & 10.67 & 51.69 \\ 678 & 10.74 & 52.25 \\ 778 & 10.95 & 51.78\end{array}$

$\begin{array}{lcc}407 & 19.26 & 34.9 \\ 404 & 19.14 & 34.6 \\ 407 & 19.09 & 34.8 \\ 381 & 19.15 & 34.9 \\ 390 & 19.12 & 35.1 \\ 345 & 19.19 & 35.1 \\ 315 & - & 35.1 \\ 286 & 19.32 & 35.2 \\ 243 & 19.41 & 35.2 \\ 268 & 19.41 & 35.2 \\ 209 & 19.32 & 35.2 \\ 169 & 19.42 & 35.2\end{array}$


Table 1. (Continued).

\begin{tabular}{|c|c|c|c|c|c|c|c|c|c|c|}
\hline $\begin{array}{c}\text { Sample } \\
\text { (interval in } \mathrm{cm} \text { ) }\end{array}$ & $\begin{array}{l}\text { Sub-bottom } \\
\text { depth (m) }\end{array}$ & $\mathrm{pH}$ & $\begin{array}{l}\text { Alkalinity } \\
\text { (meq/liter) }\end{array}$ & $\begin{array}{l}\mathrm{SO}_{4}{ }^{2-} \\
(\mathrm{mM})\end{array}$ & $\frac{\mathrm{SiO}_{2}}{(\mu M)}$ & $\begin{array}{l}\mathrm{Ca}^{2+} \\
(\mathrm{m} M)\end{array}$ & $\begin{array}{l}\mathrm{Mg}^{2+} \\
(\mathrm{m} M)\end{array}$ & $\begin{array}{l}\mathrm{Sr}^{2+} \\
(\mu M)\end{array}$ & $\begin{array}{c}\mathrm{Cl} \\
\left(\% \%_{0}\right)\end{array}$ & $\underset{(\%)}{S}$ \\
\hline \multicolumn{11}{|l|}{ Hole 573B (Cont.) } \\
\hline $36-1,135-150$ & 472 & 7.47 & 2.85 & 25.6 & 762 & 10.59 & 52.74 & 156 & 19.40 & 35.4 \\
\hline $39-2,135-150$ & 502 & 7.45 & 2.50 & 25.5 & 724 & 10.04 & 53.02 & 117 & 19.49 & 35.1 \\
\hline \multicolumn{11}{|l|}{ Hole 574} \\
\hline $1-1,144-150$ & 1.5 & 7.45 & 2.61 & 28.7 & 599 & 10.33 & 53.03 & 93 & 19.24 & 35.2 \\
\hline $2-5,144-150$ & 12 & 7.44 & 2.78 & 27.8 & 692 & 10.59 & 52.94 & 123 & 19.31 & 35.2 \\
\hline $3-4,144-150$ & 20 & 7.44 & 2.91 & 28.8 & 732 & 10.70 & 53.10 & 131 & 19.34 & 35.4 \\
\hline $4-5,144-150$ & 31 & 7.44 & 2.77 & - & 620 & 10.76 & 52.93 & 147 & 19.49 & 35.5 \\
\hline $5-4,144-150$ & 39 & 7.40 & 2.78 & 27.1 & 620 & 10.81 & 52.60 & 154 & 19.49 & 35.4 \\
\hline $8-4,144-150$ & 67 & 7.42 & 2.76 & 27.4 & 692 & 10.50 & 51.80 & 199 & 19.38 & 35.2 \\
\hline $11-5,140-150$ & 97 & 7.33 & 3.27 & 25.7 & 732 & 10.70 & 51.04 & 230 & 19.43 & 35.2 \\
\hline $18-2,140-150$ & 141 & 7.30 & 3.46 & 25.7 & 742 & 11.01 & 50.32 & 301 & 19.30 & 35.2 \\
\hline $24-2,140-150$ & 171 & 7.27 & 3.23 & - & 770 & 11.17 & 49.66 & 327 & 19.20 & 35.2 \\
\hline $28-2,140-150$ & 199 & 7.27 & 3.54 & 25.2 & 686 & 12.04 & 49.81 & 365 & 19.25 & 35.2 \\
\hline
\end{tabular}

Hole 574A

$2-5,144-150$
$4-5,144-150$
$7-4,144-150$
$10-5,144-150$
$18-6,140-150$
$22-5,144-150$

$\begin{array}{rr}8 & 7.39 \\ 32 & 7.38 \\ 60 & 7.44 \\ 87 & 7.37 \\ 141 & 7.29 \\ 175 & 7.33\end{array}$

2.74
2.81
2.84
3.03
3.37
3.61

$\begin{array}{ccc}- & 714 & 10.92 \\ 27.8 & 735 & 11.01 \\ \overline{26.5} & 780 & 10.80 \\ - & 809 & 11.04 \\ - & 771 & 11.19 \\ & 770 & 11.17\end{array}$

52.41

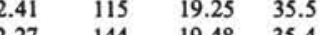
$\begin{array}{llll}51.55 & 189 & 19.38 & 35.4\end{array}$ $\begin{array}{llll}51.16 & 237 & 19.32 & 35.2 \\ 49.99 & 305 & 19.22 & 35.2\end{array}$

Hole 574B

$1-5,144-150$

$193 \quad 7.30$

3.57

25.3

859

11.80

49.66

$\begin{array}{ll}19.17 & 35.1\end{array}$

Hole 574C

$1-3,144-150$
$4-2,144-150$
$7-5,144-150$
$11-1,140-150$
$13-4,140-150$
$16-2,140-150$
$20-3,140-150$
$22-2,140-150$
$26-2,140-150$
$29-1,135-150$
$31-1,135-150$
$35-1,135-150$

$\begin{array}{ll}199 & 7.29 \\ 226 & 7.28 \\ 259 & 7.26 \\ 291 & 7.21 \\ 314 & 7.31 \\ 340 & 7.28 \\ 379 & 7.32 \\ 397 & 7.36 \\ 435 & 7.46 \\ 462 & 7.44 \\ 481 & 7.37 \\ 519 & 7.46\end{array}$

$\begin{array}{ll}3.38 & - \\ 3.48 & 25.1 \\ 3.73 & 24.0 \\ 3.83 & 24.6 \\ 3.69 & 24.5 \\ 3.43 & 24.5 \\ 3.44 & 24.3 \\ 3.17 & 25.7 \\ 1.58 & 25.5 \\ 2.56 & 26.2 \\ 2.98 & 26.8 \\ 2.45 & 25.7\end{array}$

$\begin{array}{ll}738 & 11.65 \\ 765 & 12.01 \\ 811 & 11.68 \\ 836 & 12.19 \\ 857 & 12.06 \\ 827 & 12.22 \\ 938 & 11.90 \\ 994 & 11.97 \\ - & 10.32 \\ 989 & 11.01 \\ 933 & 10.90 \\ 470 & 10.43\end{array}$

49.20
48.89
48.89
48.82
48.47
48.12
48.73
48.76
49.04
50.26
51.70
50.58

$\begin{array}{rll}346 & 19.21 & 35.2 \\ 375 & 19.21 & 35.3 \\ 378 & 19.19 & 35.2 \\ 391 & 19.19 & 35.2 \\ 384 & 19.19 & 35.2 \\ 378 & 19.17 & 35.2 \\ 344 & 19.26 & 35.2 \\ 324 & 19.32 & 35.2 \\ 249 & 19.41 & 35.2 \\ 211 & 19.46 & 35.2 \\ 173 & 19.46 & 35.8 \\ 93 & 19.04 & 34.9\end{array}$

Hole 575

$1-1,144-150$
$2-2,144-150$
$3-5,144-150$
$4-5,144-150$
$5-5,144-150$
$7-5,144-150$
$10-4,144-150$

$\begin{array}{cc}1.5 & 7.44 \\ 10 & 7.41 \\ 24 & 7.34 \\ 34 & 7.25 \\ 43 & 7.34 \\ 61 & 7.30 \\ 86 & 7.26\end{array}$

7.44
7.41
7.34
7.25
7.34
7.30
7.26

2.61
2.62
2.81
2.96
2.96
3.14
3.21

27.9
28.2
29.0
26.7
26.4
25.1
25.9

\begin{tabular}{ll}
- & 10.78 \\
$=$ & 10.64 \\
- & 10.62 \\
- & 10.60 \\
- & 10.84 \\
- & 11.14 \\
\hline & 11.55
\end{tabular}

52.35

52.85

52.75

51.94

51.37

ole 575A

$1-1,144-150$
$5-3,140-150$
$10-2,140-150$
$18-2,140-150$
$26-3,140-150$
$30-2,140-150$
$33-2,140-150$

$\begin{array}{rr}95 & 7.35 \\ 114 & 7.28 \\ 130 & 7.34 \\ 155 & 7.33 \\ 185 & 7.25 \\ 197 & 7.29 \\ 207 & 7.20\end{array}$

3.11
3.27
3.18
3.25
3.17
3.52
3.58

25.6
25.5
25.2
26.0
24.9
25.0
24.5

-
$\overline{-}$
$\overline{-}$
-

11.77
11.94
11.84
12.28
11.93
12.64
12.20

$\begin{array}{llll}49.74 & 259 & 19.24 & 35.2 \\ 49.26 & 285 & 19.16 & 35.2 \\ 49.16 & 294 & 19.17 & 35.2 \\ 48.74 & 311 & 19.21 & 35.2 \\ 48.40 & 347 & 19.20 & 34.9 \\ 48.14 & 357 & 19.14 & 34.9 \\ 48.10 & 374 & 19.18 & 35.1\end{array}$

Hole 575B

$\begin{array}{lrlllllllll}1-1,144-150 & 5 & 7.39 & 2.64 & 28.3 & - & 10.41 & 52.26 & 101 & 19.10 & 35.2 \\ 6-4,144-150 & 54 & 7.39 & 2.91 & 26.4 & - & 10.87 & 51.75 & 181 & 19.43 & 35.2 \\ 13-2,140-150 & 112 & 7.34 & 3.25 & 26.3 & - & 11.76 & 49.77 & 273 & 19.17 & 35.2\end{array}$

Note: - indicates that sample was not analyzed. 
Table 2. Thicknesses of sedimentary zones divided on the basis of dissolved- $\mathrm{Sr}^{2}+$ profiles.

\begin{tabular}{cccc}
\hline Site & $\begin{array}{c}\text { Zone I } \\
(\mathrm{m})\end{array}$ & $\begin{array}{c}\text { Zone II } \\
(\mathrm{m})\end{array}$ & $\begin{array}{c}\text { Zone III } \\
(\mathrm{m})\end{array}$ \\
\hline 572 & $0-260$ & $260-360$ & $360-480$ \\
573 & $0-110$ & $110-270$ & $270-520$ \\
574 & $0-200$ & $200-340$ & $340-510$ \\
\hline
\end{tabular}

Note: Zone $\mathrm{I}=$ depth interval of increasing dissolved $\mathrm{Sr}^{2}+$ concentrations. Zone II $=$ depth interval of approximately constant maximum dissolved $\mathrm{Sr}^{2+}$ concentrations. Zone III = Depth interval of decreasing dissolved $\mathrm{Sr}^{2+}$ concentrations.

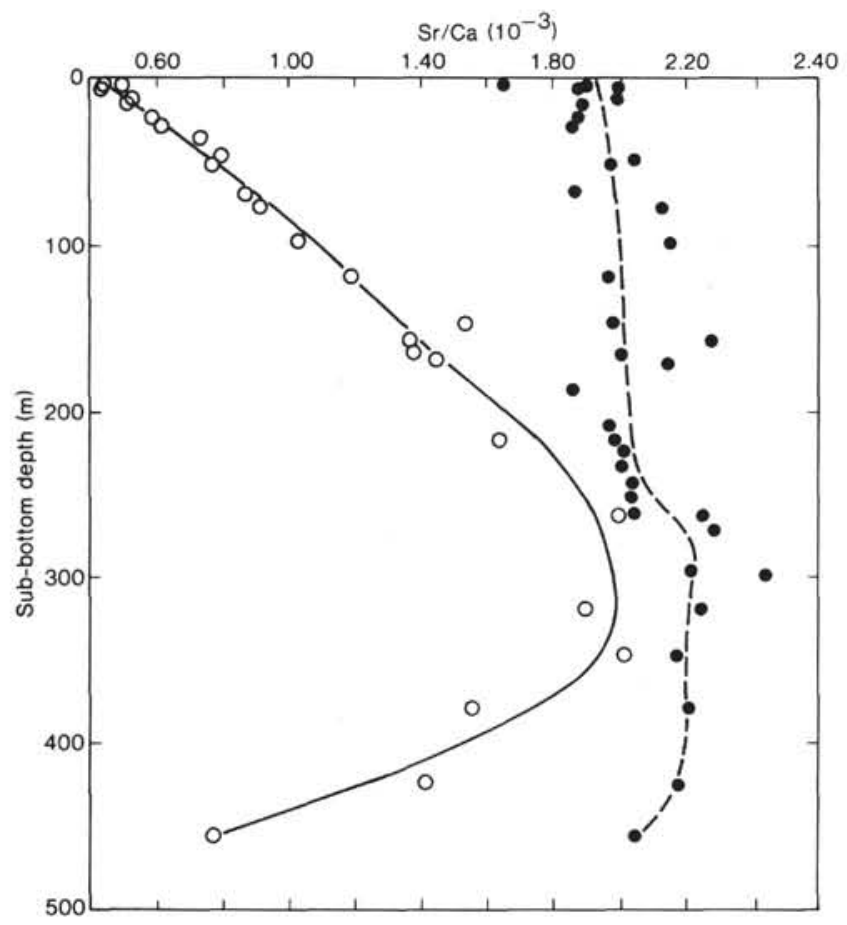

Figure 7. Calculated equilibrium $\mathrm{Sr} / \mathrm{Ca}$ ratios $(\mathrm{O})$ and observed ratios (•) for bulk carbonates for Site 572 .

sils have higher strontium concentrations in their skeletal calcite than foraminifers.

Regarding the second factor, studies have suggested that the $\mathrm{Sr} / \mathrm{Ca}$ ratio of seawater varies with age (Graham et al., 1982). Such chemical changes could influence the strontium distribution in biogenic calcite. Graham et al. (1982) presented data on variations in $\mathrm{Sr} / \mathrm{Ca}$ ratios in planktonic foraminifers extending back to the Mesozoic. They suggested that the observed changes in $\mathrm{Sr} / \mathrm{Ca}$ ratios with respect to geologic age resulted from basin-to-shelf fractionation between $\mathrm{Sr}$-rich aragonite and less Sr-rich calcite. They suggested that the age differences may also reflect changing calcium fluxes varying in response to different rates of seafloor spreading. Cronblad and Malmgren (1981) showed that in Quaternary sediments, the strontium concentrations of two forami-

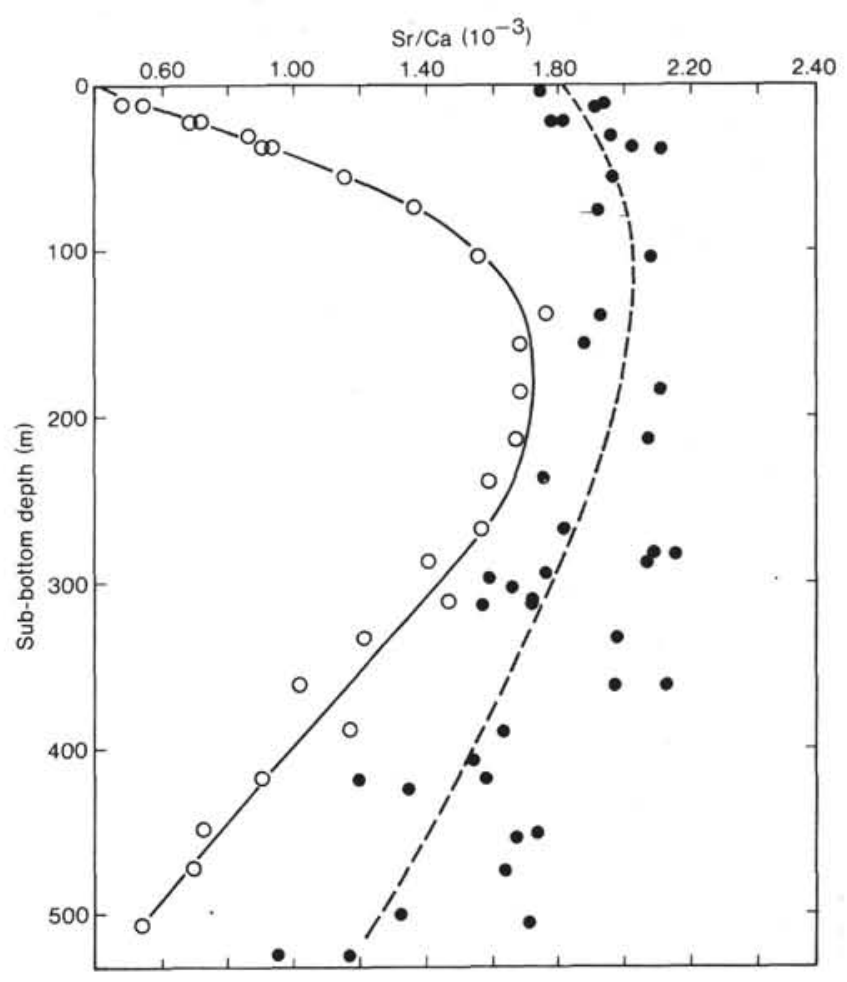

Figure 8. Calculated equilibrium $\mathrm{Sr} / \mathrm{Ca}$ ratios $(\mathrm{O})$ and observed ratios (•) for bulk carbonates for Site 573 .

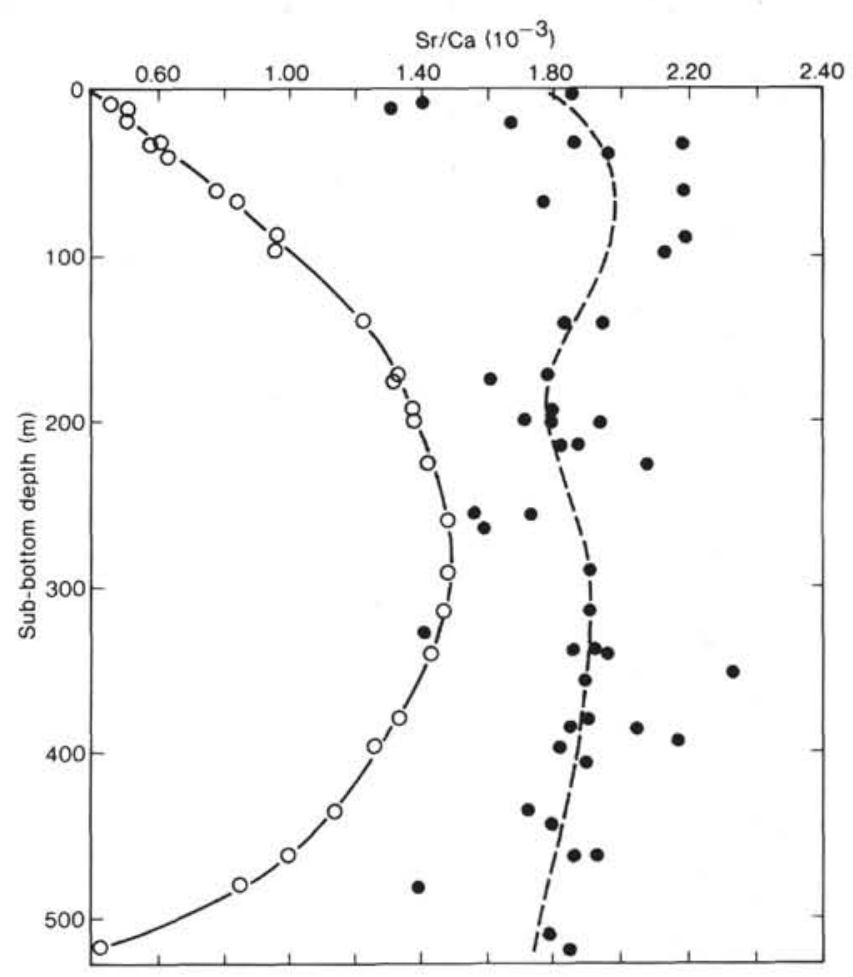

Figure 9. Calculated equilibrium $\mathrm{Sr} / \mathrm{Ca}$ ratios $(O)$ and observed ratios (•) for bulk carbonates for Site 574 . 
Table 3. $\mathrm{Sr} / \mathrm{Ca}$ ratios of carbonate samples and calculated equilibrium $\mathrm{Sr} / \mathrm{Ca}$ ratios derived from interstitial water profiles.

\begin{tabular}{lcccc}
\hline $\begin{array}{c}\text { Sample } \\
\text { (interval in cm) }\end{array}$ & $\begin{array}{c}\text { Sub-bottom } \\
\text { depth (m) }\end{array}$ & $\begin{array}{c}\text { Age } \\
(\mathrm{Ma})\end{array}$ & $\begin{array}{c}\mathrm{Sr} / \mathrm{Ca} \\
\left(10^{3}\right)\end{array}$ & $\begin{array}{c}\mathrm{Sr} / \mathrm{Ca} q \\
\left(10^{3}\right)\end{array}$ \\
\hline Hole 572 & & & & \\
& & & & \\
1-1, 115-135 & 1 & 0.1 & 1.90 & 0.40 \\
1-2, 100-120 & 2.5 & 0.2 & 1.99 & 0.44 \\
1-3, 100-120 & 4 & 0.2 & 1.88 & 0.50 \\
Hole 572A & & & & \\
& & & & \\
1-1, 0-20 & 0.5 & 0.1 & 1.65 & 0.41 \\
1-4, 50-70 & 5 & 0.3 & 1.88 & 0.44 \\
2-1, 0-20 & 9.5 & 0.6 & 1.99 & 0.53 \\
3-2, 115-135 & 22 & 1.5 & 1.87 & 0.59 \\
4-4, 95-115 & 34 & 2.2 & & 0.74 \\
6-3, 85-105 & 50 & 3.0 & 1.97 & 0.78 \\
$9-3,85-105$ & 76 & 4.4 & 2.12 & 0.92 \\
13-5, 85-105 & 117 & 5.2 & 1.97 & 1.19
\end{tabular}

Hole 572B
$2-3,123-143$
164
$6.1 \quad 2.01$
1.38

Hole $572 \mathrm{C}$

$\begin{array}{lrrrr}2-2,144-150 & 13 & 0.8 & 1.89 & 0.51 \\ 3-5,144-150 & 27 & 1.9 & 1.86 & 0.62 \\ 5-5,144-150 & 46 & 2.8 & 2.04 & 0.80 \\ 7-5,144-150 & 66 & 4.1 & 1.87 & 0.88 \\ 10-5,144-150 & 97 & 4.8 & 2.15 & 1.03 \\ 17-3,144-150 & 145 & 5.7 & 1.98 & 1.54\end{array}$

Hole 572D

$\begin{array}{llrlc}1-3,144-150 & 155 & 5.9 & 2.27 & 1.37 \\ 2-5,144-150 & 168 & 6.2 & 2.14 & 1.45 \\ 4-4,78-80 & 185 & 6.5 & 1.86 & - \\ 6-6,80-82 & 207 & 6.9 & 1.97 & - \\ 7-5,144-150 & 215 & 7.5 & 1.99 & 1.64 \\ 8-3,100-102 & 222 & 8.0 & 2.01 & - \\ 9-3,100-102 & 231 & 8.7 & 2.00 & - \\ 10-3,100-102 & 241 & 9.4 & 2.04 & - \\ 11-3,100-102 & 250 & 10.1 & 2.03 & - \\ 12-3,100-102 & 260 & 10.8 & 2.04 & - \\ 12-4,144-150 & 261 & 10.8 & 2.24 & 2.00 \\ 13-3,100-102 & 269 & 11.5 & 2.28 & - \\ 16-1,58-60 & 294 & 12.0 & 2.22 & - \\ 16-3,100-102 & 298 & 12.1 & 2.44 & - \\ 18-4,144-150 & 318 & 12.3 & 2.24 & 1.90 \\ 21-4,144-150 & 346 & 12.7 & 2.18 & 2.02 \\ 24-5,144-150 & 377 & 13.3 & 2.21 & 1.56 \\ 29-4,140-150 & 423 & 14.1 & 2.18 & 1.42 \\ 32-4,140-150 & 454 & 14.6 & 2.05 & 0.78\end{array}$

Hole 573

$\begin{array}{lcccc}1-4,144-150 & 1.5 & 0.2 & 1.74 & 0.39 \\ 2-6,144-150 & 11 & 0.7 & 1.91 & 0.55 \\ 3-6,144-150 & 20.5 & 1.3 & 1.78 & 0.72 \\ 5-5,144-150 & 38 & 2.6 & 2.11 & 0.94 \\ 7-5,140-150 & 55 & 3.9 & 1.97 & 1.15 \\ 9-5,140-150 & 74 & 4.9 & 1.92 & 1.37 \\ 12-5,140-150 & 103 & 6.1 & 2.08 & 1.56 \\ 16-5,140-150 & 138 & 8.5 & 1.93 & 1.77\end{array}$

Hole $573 \mathrm{~A}$

$\begin{array}{lllll}1-4,144-150 & 10.5 & 0.7 & 1.94 & 0.48 \\ 2-4,143-150 & 21 & 1.3 & 1.82 & 0.69 \\ 3-5,143-150 & 30 & 2.0 & 1.96 & 0.86 \\ 4-4,143-150 & 37 & 2.6 & 2.03 & 0.91 \\ 6-4,143-150 & 55 & 3.9 & & 1.16\end{array}$

Hole 573B

\begin{tabular}{lrrrr}
$2-5,143-150$ & 156 & 9.7 & 1.88 & 1.69 \\
$5-5,144-150$ & 184 & 12.4 & 2.11 & 1.69 \\
$8-5,140-150$ & 212 & 13.7 & 2.08 & 1.68 \\
$11-4,140-150$ & 239 & 15.0 & 1.75 & 1.59 \\
\hline
\end{tabular}

Table 3. (Continued).

\begin{tabular}{ccccc}
\hline $\begin{array}{c}\text { Sample } \\
\text { (interval in cm) }\end{array}$ & $\begin{array}{c}\text { Sub-bottom } \\
\text { depth (m) }\end{array}$ & $\begin{array}{c}\text { Age } \\
\text { (Ma) }\end{array}$ & $\begin{array}{c}\mathrm{Sr} / \mathrm{Ca} \\
\left(10^{3}\right)\end{array}$ & $\begin{array}{c}\text { Sr/Caequil } \\
\left(10^{3}\right)\end{array}$ \\
\hline Hole 573B (Cont.) & & & & \\
$14-4,140-150$ & 268 & 17.7 & 1.82 & 1.57 \\
$16-2,10-12$ & 283 & 19.2 & 2.09 & - \\
$16-2,49-51$ & 283 & 19.2 & 2.15 & - \\
$16-4,140-150$ & 287 & 19.7 & 2.07 & 1.41 \\
$17-3,51-53$ & 294 & 20.4 & 1.76 & - \\
$17-5,68-70$ & 297 & 20.7 & 1.59 & - \\
$19-2,65-95$ & 312 & 21.8 & 1.72 & 1.47 \\
$21-3,140-150$ & 333 & 23.3 & 1.98 & 1.22 \\
$24-3,140-150$ & 361 & 26.7 & 1.99 & 1.06 \\
$27-2,140-150$ & 388 & 28.8 & 1.63 & 1.17 \\
$30-2,140-150$ & 417 & 30.8 & 1.20 & 0.91 \\
$33-5,140-150$ & 450 & 32.2 & 1.73 & 0.73 \\
$36-1,140-150$ & 472 & 33.2 & 1.64 & 0.70 \\
$39-2,135-150$ & 502 & 34.5 & 1.71 & 0.55 \\
$42-2,30-32$ & 522 & 37.0 & 2.19 & - \\
$42-3,74-76$ & 525 & 37.3 & 7.98 & - \\
$43-3,128-130$ & 526 & 37.8 & 1.16 & -
\end{tabular}

Hole 574

$\begin{array}{lcrll}1-1,144-150 & 1.5 & 0.2 & 1.85 & 0.40 \\ 2-5,144-150 & 12 & 2.2 & 1.31 & 0.51 \\ 3-4,144-150 & 20 & 3.5 & 1.67 & 0.51 \\ 4-5,144-150 & 31 & 4.8 & 2.18 & 0.61 \\ 5-4,144-150 & 39 & 5.4 & 1.96 & 0.63 \\ 8-4,144-150 & 67 & 9.0 & 1.76 & 0.84 \\ 11-5,140-150 & 97 & 12.1 & 2.13 & 0.96 \\ 18-2,140-150 & 141 & 13.4 & 1.84 & 1.23 \\ 24-2,144-150 & 171 & 16.0 & 1.78 & 1.33 \\ 28-2,140-150 & 199 & 17.0 & 1.71 & 1.38\end{array}$

Hole $574 \mathrm{~A}$

$\begin{array}{lrrrr}2-5,144-150 & 8 & 1.4 & 1.40 & 0.46 \\ 4-5,144-150 & 32 & 4.9 & 1.86 & 0.58 \\ 7-4,144-150 & 60 & 6.7 & 2.18 & 0.78 \\ 10-5,144-150 & 87 & 11.3 & 2.19 & 0.96 \\ 18-6,144-150 & 141 & 13.4 & 1.84 & 1.23 \\ 22-5,144-150 & 175 & 16.2 & 1.60 & 1.32\end{array}$

Hole 574B

$\begin{array}{lllll}1-5,144-150 & 193 & 16.8 & 1.79 & 1.38\end{array}$

Hole $574 \mathrm{C}$

\begin{tabular}{lllll}
$1-3,144-155$ & 199 & 17.0 & 1.79 & 1.38 \\
$1-5,37-39$ & 201 & 17.0 & 1.94 & - \\
$3-1,126-128$ & 215 & 17.5 & 1.87 & - \\
$3-2,6-8$ & 215 & 17.5 & 1.81 & - \\
$4-2,140-150$ & 226 & 17.8 & 2.08 & 1.42 \\
$7-5,144-150$ & 259 & 18.9 & 1.73 & 1.48 \\
$11-1,140-150$ & 291 & 20.1 & 1.92 & 1.48 \\
$13-4,140-150$ & 314 & 21.2 & 1.91 & 1.47 \\
$16-1,71-73$ & 338 & 22.3 & 1.86 & - \\
$16-1,95-97$ & 338 & 22.3 & 1.92 & - \\
$16-2,140-150$ & 340 & 22.5 & 1.96 & 1.43 \\
$17-4,120-122$ & 352 & 24.0 & 2.33 & - \\
$17-4,124-126$ & 352 & 24.0 & 2.33 & - \\
$18-2,54-56$ & 358 & 24.8 & 1.89 & - \\
$20-3,140-150$ & 379 & 27.1 & 1.91 & 1.34 \\
$21-1,60-62$ & 385 & 27.6 & 2.05 & - \\
$21-1,50-52$ & 385 & 27.6 & 1.85 & - \\
$22-1,19-21$ & 394 & 28.3 & 2.18 & - \\
$22-2,140-150$ & 397 & 28.7 & 1.81 & 1.26 \\
$23-3,82-84$ & 407 & 29.4 & 1.89 & - \\
$26-2,140-150$ & 435 & 31.6 & 1.72 & 1.14 \\
$27-2,75-77$ & 444 & 32.4 & 1.80 & \\
$29-1,135-150$ & 462 & -33.9 & 1.93 & 0.90 \\
$29-2,56-58$ & 462 & 33.9 & 1.86 & \\
$31-1,135-150$ & 481 & 35.1 & 1.40 & 0.75 \\
$34-1,120-122$ & 509 & - & 1.99 & \\
$35-1,135-140$ & 519 & 37.5 & 2.03 & 0.42 \\
\hline
\end{tabular}

Note: - indicates that no interstitial water sample was taken at this depth. 
niferal species co-varied with paleoclimatic indices. They claimed that changes in the temperature of seawater accounted for the strontium variations.

The third important factor, the extent and the physicochemical conditions of recrystallization during diagenesis, also contributes to variations in $\mathrm{Sr} / \mathrm{Ca}$ ratios in the carbonates. Large intervals of closely spaced, interlayered chalks and oozes were observed throughout Leg 85 cores and have been reported in many deep-sea carbonate studies (e.g., Schlanger and Douglas, 1974; Garrison, 1981). These differences imply that calcite recrystallization occurs more rapidly in some layers than in others. Suggested explanations include preburial halmyrolitic and early diagenetic influences, such as variations in sedimentation rates and the amount of dissolution of labile species at the benthic boundary layer. Postburial diagenetic processes can also vary. For example, experimental evidence (deep-sea sediments reacting at elevated temperatures) indicate that the amount of clay minerals and biogenic silica may affect the rate of calcite recrystallization (Baker et al., 1980).

I now discuss how these different factors may account for the observed trends from Leg 85 sites and compare these with results from other DSDP sites drilled in the equatorial Pacific. I also present an estimate of the amount of calcite recrystallization that has occurred at Sites 572, 573, and 574 .

\section{VARIATIONS IN CALCAREOUS MICROFOSSIL COMPOSITION}

I examined the relationship between the $\mathrm{Sr} / \mathrm{Ca}$ ratios measured for the bulk carbonates and the calcareous microfossil percentages of adjacent sediments, which are taken from shipboard smear-slide results. The $\mathrm{Sr} / \mathrm{Ca}$ ratios are compared with the nannofossil/foraminifer ratios. With the exception of three samples from Site 574, all samples contained more nannofossils than foraminifers. The results are summarized in Table 4 . The average $\mathrm{Sr} / \mathrm{Ca}$ ratios, the mean nannofossil/foraminifer ratios, and a correlation coefficient $(r)$ for a linear regression analysis of the data are presented. Values of these parameters are given for each of the three zones (as defined above and described in Table 2), which divide the sediment section according to trends in the dissolved

Table 4. $\mathrm{Sr} / \mathrm{Ca}$ ratios in bulk carbonates versus calcareous microfossil contents.

\begin{tabular}{lcccrc}
\hline Site & Zone & $\begin{array}{c}\text { Mean Sr/Ca } \\
\left(10^{3}\right)\end{array}$ & $\begin{array}{c}\text { Mean } \\
\log \mathrm{N} / \mathrm{F}^{\mathrm{a}}\end{array}$ & & $\begin{array}{c}\text { Sr/Ca nanno } \\
\left(10^{3}\right)\end{array}$ \\
\hline 572 & I & $1.96 \pm 0.13$ & $1.01 \pm 0.5$ & 0.765 & 2.35 \\
& II & $2.01 \pm 0.03$ & $1.77 \pm 0.1$ & 0.525 & \\
573 & III & $2.01 \pm 0.10$ & $1.22 \pm 0.6$ & 0.489 & \\
& I & $1.93 \pm 0.12$ & $0.81 \pm 0.5$ & 0.613 & 2.43 \\
& II & $1.93 \pm 0.14$ & $1.74 \pm 0.3$ & 0.189 & \\
574 & III & $1.77 \pm 0.31$ & $1.26 \pm 0.6$ & 0.153 & \\
& I & $1.82 \pm 0.3$ & $0.74 \pm 0.7$ & 0.823 & 2.34 \\
& II & $1.84 \pm 0.12$ & $1.71 \pm 0.7$ & 0.579 & \\
& III & $1.92 \pm 0.19$ & $1.44 \pm 0.3$ & -0.052 & \\
\hline
\end{tabular}

\footnotetext{
${ }^{a} \log N / F=\log \frac{\% \text { Nannofossils }}{\% \text { Foraminifers }}$

${ }^{b} \mathrm{Sr}_{\mathrm{C}} / \mathrm{Ca}$ nanno $=$ extrapolated value, assuming sediments composed of $100 \%$ nannofossils.
}

strontium profiles. The best positive correlation coefficient for each site occurs in Zone I, which is the depth interval for which the $\mathrm{Sr} / \mathrm{Ca}$ distributions between observed and predicted values suggest that calcite recrystallization has been minor. An extrapolated value for the $\mathrm{Sr} / \mathrm{Ca}$ ratios, corresponding to a sample composed exclusively of nannofossils, is presented for this upper zone (Table 4). All three regression analyses extrapolate to a similar value, and this $\mathrm{Sr} / \mathrm{Ca}$ ratio is slightly higher than any observed ratio, except for two samples. These results suggest that much of the $\mathrm{Sr} / \mathrm{Ca}$ variations in carbonates from Zone I can be attributed to different proportions of calcareous microfossils, and they support the implication that nannofossils incorporate more strontium in their skeletal structures than foraminifers. The lower values for the correlation coefficients for Zones II and III suggest that the $\mathrm{Sr} / \mathrm{Ca}$ variations are controlled largely by calcite recrystallization and, to a much lesser extent, by microfossil content.

\section{CHEMICAL OCEANOGRAPHIC FACTORS}

$\mathrm{The} \mathrm{Sr} / \mathrm{Ca}$ ratios for all sediment samples from Leg 85 are plotted versus age in Figure 10. Also plotted are data from Site 289, drilled on the Ontong-Java Plateau, reported by Elderfield et al. (1982). Some of the large differences observed in $\mathrm{Sr} / \mathrm{Ca}$ values from samples of similar ages result from variations in calcareous microfossil content. The low values at 1.4, 2.7, and 3.5 Ma for Site 574 and the overall lower ratios for Sites 575 and 289 are attributable to higher foraminifer contents. Despite these differences, there appears to be an age control on the $\mathrm{Sr} / \mathrm{Ca}$ ratios, at least from early Miocene to the Recent. Older sediments are more extensively recrystallized. The most important fact to note in Figure 10 is that all curves have relatively low $\mathrm{Sr} / \mathrm{Ca}$ ratios for sediments deposited during the late Miocene and near the boundary of early/middle Miocene and relatively higher $\mathrm{Sr} / \mathrm{Ca}$ ratios for middle Miocene sediments. These results resemble those of Graham et al. (1982) for planktonic foraminifers. These data also help explain at least some of the variations in $\mathrm{Sr} / \mathrm{Ca}$ ratios in Zone I of the three sites studied (Figs. 7 to 9; Tables 3 and 4). In addition, some of the observed variations in the older, more extensively recrystallized sediments in Zones II and III may reflect primary differences in the calcareous deposits-differences that were controlled by paleochemical fluctuations in seawater $\mathrm{Sr} / \mathrm{Ca}$ ratios. In principle, diagenetic reactions do not necessarily obliterate paleoceanographic signals, although they undoubtedly modify them.

\section{VARIATIONS IN CARBONATE DIAGENESIS}

$\mathrm{The} \mathrm{Sr} / \mathrm{Ca}$ ratios in the carbonates from Sites 572, 573, and 574 differ in overall trend and in the extent of divergence from equilibrium ratios within the depth interval where calcite recrystallization is apparent (Zones II and III) (Figs. 7 to 9; Tables 3 and 4). These data indicate differences in diagenetic histories among the three sites. The average $\mathrm{Sr} / \mathrm{Ca}$ ratios in carbonates from Sites 572 and 574 increase slightly with depth, whereas the ratios decrease with depth at Site 573. There is also less 
scatter in observed $\mathrm{Sr} / \mathrm{Ca}$ values from Sites 572 and 574 as compared with Site 573, especially in Zone III. Site 573 is also unique in that a general trend of observed $\mathrm{Sr} / \mathrm{Ca}$ ratios approaching equilibrium values with depth is apparent (Fig. 8).

At Site 572, there seems to be a correlation between the carbonate $\mathrm{Sr} / \mathrm{Ca}$ ratios and the carbonate accumulation rates. The high $\mathrm{Sr} / \mathrm{Ca}$ ratios, which approach a constant value of $2.2 \times 10^{-3}$ in the depth interval below $260 \mathrm{~m}$ (Fig. 7), coincide with large carbonate mass accumulation rates, ranging from 2.4 to $5.0 \mathrm{~g} / \mathrm{cm}^{2}$ per thousand years as determined on board ship. More solutionsusceptible species of calcareous microfossils and more organic material are buried at times of high accumulation rates. This might enhance diagenetic reactions because the biogenic calcite might dissolve at faster rates. The higher $\mathrm{Sr} / \mathrm{Ca}$ ratios in the solids at this site may thus reflect greater rates of strontium production, which in turn generates more dissolved $\mathrm{Sr}^{2+}$ in interstitial waters. Consequently, more strontium is incorporated into the inorganically produced calcite. Carbonate accumulation rates were lower at Sites 573 and 574, and the overall $\mathrm{Sr} / \mathrm{Ca}$ ratios are not as high as those at Site $\mathbf{5 7 2}$ (Figs. 7 to 9; Tables 3 and 4). Also, a higher temperature gradient at Site 572 may have contributed to the higher $\mathrm{Sr} / \mathrm{Ca}$ values in these sediments.

$\mathrm{The} \mathrm{Sr} / \mathrm{Ca}$ ratios from intervals containing closely spaced ooze and chalk layers from Sites 573 and 574 were determined. Care was taken when sampling to ensure that the induration variations were not artifacts of drilling. The data are summarized in Table 5. The chalks were significantly lower than the oozes in $\mathrm{Sr} / \mathrm{Ca}$ concentrations in only two of the intervals studied.

The disagreement between the predicted and observed $\mathrm{Sr} / \mathrm{Ca}$ distributions in Zone III at each site illustrates a problem inherent in comparing present interstitial water data with measurements from adjacent solids. The $\mathrm{Sr} /$ $\mathrm{Ca}$ values in solids from these deeper intervals most probably reflect recrystallization at times when pore water concentrations were significantly different from those at present. The higher $\mathrm{Sr} / \mathrm{Ca}$ ratios of the carbonates versus the equilibrium values in Zone III may represent recrystallization that occurred in the past when dissolved $\mathrm{Sr}^{2+}$ concentrations were higher. The predominance of chalk in sediments from this zone indicates that carbonate diagenesis has already been significant (Fig. 2). The decreasing, roughly linear, dissolved strontium gradient in Zone III suggests that the pore water profile repre-

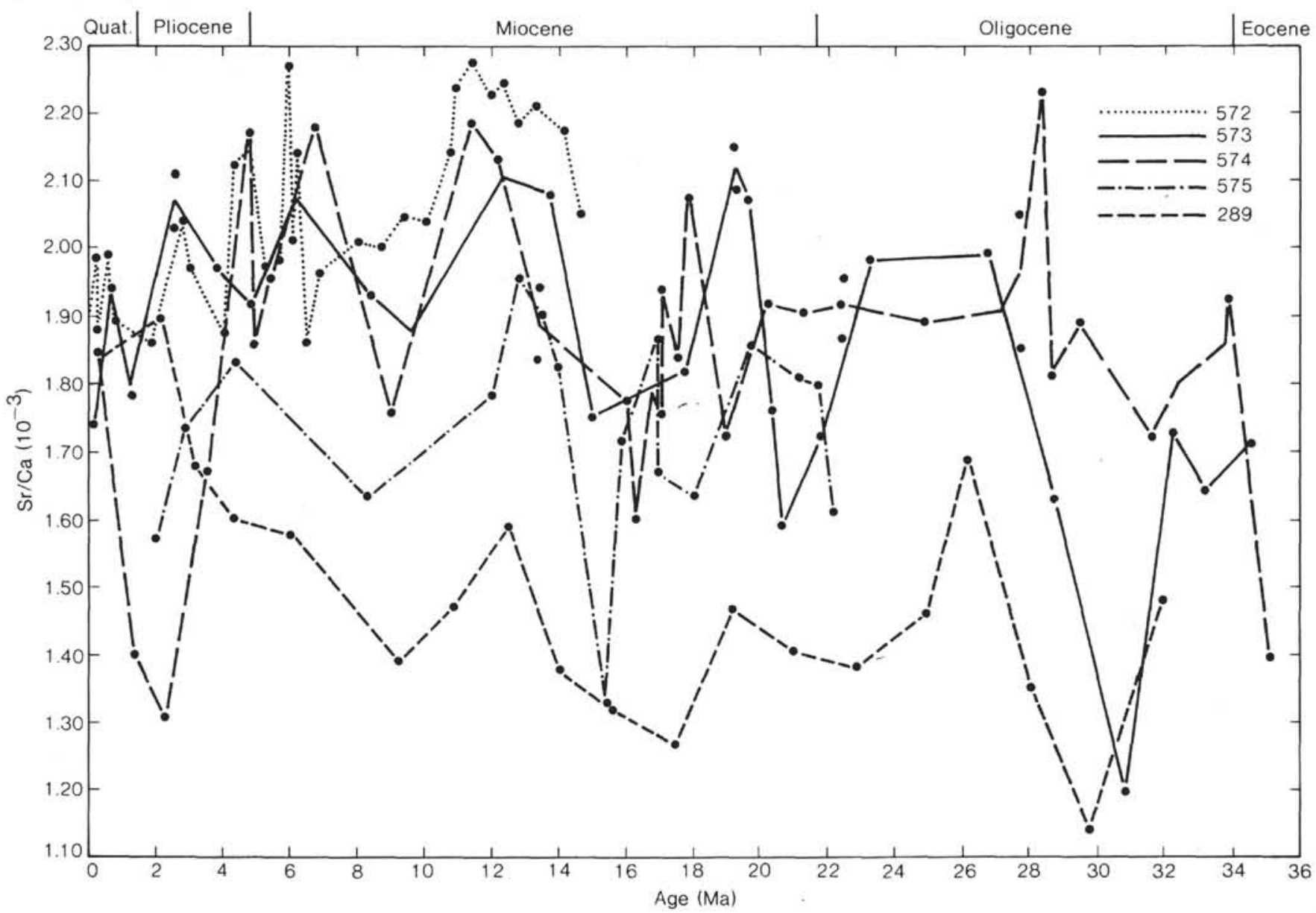

Figure 10. Sr/Ca ratios of bulk carbonates versus age for DSDP sites from the equatorial Pacific. Site 289 data are from Elderfield et al. (1982) for sediments from the Ontong-Java plateau, Leg 30. 
Table 5. Sr/Ca ratios in pairs of adjacent chalk and ooze layers.

\begin{tabular}{|c|c|c|}
\hline $\begin{array}{c}\text { Sample } \\
\text { (interval in } \mathrm{cm} \text { ) }\end{array}$ & $\begin{array}{l}\mathrm{Sr} / \mathrm{Ca} \\
\left(10^{3}\right)\end{array}$ & $\begin{array}{c}\text { Sedimen } \\
\text { type }\end{array}$ \\
\hline \multicolumn{3}{|l|}{ Hole 573B } \\
\hline $16-2,10-12$ & 2.09 & Ooze \\
\hline $16-2,49-51$ & 2.15 & Chalk \\
\hline $17-3,51-53$ & 1.76 & Ooze \\
\hline $17-5,6-8$ & 1.59 & Chalk \\
\hline \multicolumn{3}{|l|}{ Hole $574 \mathrm{C}$} \\
\hline $3-1,136-137$ & 1.87 & Ooze \\
\hline $3-2,6-7$ & 1.81 & Chal \\
\hline $16-1,95-97$ & 1.92 & Ooze \\
\hline $16-1,71-72$ & 1.86 & Chal \\
\hline $17-4,120-121$ & 2.33 & Ooze \\
\hline $17-4,124-125$ & 2.33 & Chal \\
\hline $21-1,60-61$ & 2.05 & Ooze \\
\hline $21-1,50-51$ & 1.85 & Chalk \\
\hline
\end{tabular}

sents diffusion downward toward the basement from overlying sediments in which recrystallization is occurring most actively at present and also suggests some strontium sink. Consequently, using the pore water $\mathrm{Sr} / \mathrm{Ca}$ values to predict "equilibrium" values for these deeper sediments may be inappropriate because processes controlling the $\mathrm{Sr}^{2+} / \mathrm{Ca}^{2+}$ distributions may be unrelated to previously recrystallized carbonates. It follows that the driving forces for continuous recrystallization and reequilibration with interstitial fluids in this zone are inadequate under existing diagenetic conditions. There is evidence, however, for some deeper diagenesis, particularly at Site 573 , where there is an overall $\mathrm{Sr} / \mathrm{Ca}$ decrease in the solids and where there is a very low value in the limestone interval $\left(\mathrm{Sr} / \mathrm{Ca}=0.95 \times 10^{-3}\right)$ at $525 \mathrm{~m}$ sub-bottom depth.

\section{EXTENT OF CALCITE RECRYSTALLIZATION}

To quantify the extent of recrystallization in the calcareous sediments from Leg 85 , a model based largely on observed strontium pore water profiles is presented. It assumes that (1) production of strontium resulting from biogenic calcite recrystallization occurs exclusively in the depth interval of maximum $\mathrm{Sr}^{2+}$ concentration (Zone II, Table 2); (2) only diffusive processes operate in Zones I and III; (3) calculated diffusive fluxes for the present have been the same in the past, since the time recrystallization initiated at each site; and (4) recrystallization did not start until a sufficiently large amount of carbonate had accumulated at each site.

Diffusive fluxes are calculated by determining diffusion coefficients and gradients from observed profiles using shipboard electrical resistivity and porosity data and appropriate temperature corrections assuming a $40^{\circ} \mathrm{C} / \mathrm{km}$ geothermal gradient (Manheim, and Waterman, 1974; McDuff and Gieskes, 1976; Berner, 1980; Baker et al., 1982). Pertinent data used for calculations, the present diffusive fluxes, the $\mathrm{Sr}^{2+}$ production rates, and the carbonate mass accumulation rates for sediments from Zones II and III are presented in Tables 6 and 7.
Table 6. Diffusion coefficients and porosities used to calculate dissolved strontium fluxes from Zone II.

\begin{tabular}{cccc}
\hline Site & Zone & $D_{\mathrm{b}}$ avg $^{\mathrm{a}}$ & $\phi$ avg $^{\mathrm{b}}$ \\
\hline 572 & I & 3.4 & 0.70 \\
& III & 3.5 & 0.63 \\
573 & I & 2.6 & 0.71 \\
& III & 3.2 & 0.52 \\
574 & I & 2.7 & 0.70 \\
& III & 2.9 & 0.53
\end{tabular}

${ }^{\mathrm{a}} D_{\mathrm{b}}$ avg $=$ average bulk diffusion boefficient $\left(10^{6} / \mathrm{cm}^{2} / \mathrm{sec}\right)$.

Table 7. Production of dissolved $\mathrm{Sr}^{2+}$ from carbonate recrystallization.

\begin{tabular}{lccccc}
\hline Site & $\begin{array}{c}\text { Time recryst. } \\
(\mathrm{Ma})\end{array}$ & $\begin{array}{c}\mathrm{J} \mathrm{Sr}^{2+\mathrm{b}} \\
\left(\mathrm{mol} / \mathrm{cm}^{2} / \mathrm{sec}\right)\end{array}$ & $\begin{array}{c}\Sigma \mathrm{Sr}^{2+\mathrm{c}} \\
\left(\mathrm{mol} / \mathrm{cm}^{2}\right)\end{array}$ & $\begin{array}{c}\text { Rate Sr}{ }^{2+\mathrm{d}} \\
\left(\mathrm{mol} / \mathrm{cm}^{3} / \mathrm{sec}\right)\end{array}$ & $\begin{array}{c}\text { Carb. accum. } \\
\text { rate } \\
\left(\mathrm{g} / \mathrm{cm}^{2}\right. \\
1000 \mathrm{yr})\end{array}$ \\
\hline 572 & 10 & $6.38 \times 10^{-17}$ & $2.56 \times 10^{-2}$ & $3.70 \times 10^{-21}$ & 2.82 \\
573 & 33.4 & $7.65 \times 10^{-17}$ & $8.58 \times 10^{-2}$ & $1.98 \times 10^{-21}$ & 1.35 \\
574 & 29.4 & $5.27 \times 10^{-17}$ & $5.45 \times 10^{-2}$ & $1.84 \times 10^{-21}$ & 1.63 \\
\hline
\end{tabular}

Time recryst. $=$ time since onset of recrystallization.

b $\mathrm{Sr}^{2+}$ recryst. = time since onset of recrystall

c $\mathrm{Sr}^{2+}=$ present strontium diffusive flux.

d $\mathrm{Rate}^{2} \mathrm{Sr}^{2+}=$ production rate of dissolved strontium from carbonates, Zones II and III.

e Carb. accum. rate = average carbonate mass accumulation rates for sediments from Zones II and III.

The onset of recrystallization at each site is assumed to have occurred at the following times: Site 572-10 $\mathrm{Ma}$; Site 573-33.4 Ma; and Site 574-29.4 Ma. These ages represent the time period during which the same amount of carbonate as that found in Zone I had accumulated at each site, according to past accumulation rates. These ages also represent the number of years over which the present diffusive flux is assumed to have operated, which is used to estimate the total dissolved strontium production. This reasoning follows from my assumption that calcite recrystallization is not occurring in Zone I. Approximately $130 \mathrm{~m}$ of sediment would have accumulated at Site 573 before recrystallization originated. This approximation is consistent with the present dissolved $\mathrm{Sr}^{2+}$ profile in that the strontium maximum there (i.e., the depth below which recrystallization occurs) is located at $110 \mathrm{~m}$ sub-bottom. Similar comparisons hold for the other sites.

Recrystallization estimates based on $\mathrm{Sr} / \mathrm{Ca}$ distributions are given in Table 8, along with estimates of the amount of chalk present in Zones II and III for each site. The percent recrystallization is a comparison between the amount of $\mathrm{Sr}^{2+}$ produced from Zones II and III since recrystallization initiated and the amount of $\mathrm{Sr}^{2+}$ available in the carbonates from these same zones. The amount of strontium available represents the total dissolved strontium produced in the interstitial waters by carbonate recrystallization plus the amount of strontium greater than the calculated equilibrium value. This is derived from the calculated equilibrium profiles, which compare the average equilibrium and the average ob- 
Table 8. Estimates of calcite recrystallization from $\mathrm{Sr} / \mathrm{Ca}$ distributions.

\begin{tabular}{cccrcc}
\hline Site & $\begin{array}{c}\sum \mathrm{CaCO}_{3}{ }^{\mathrm{a}} \\
(\mathrm{mol})\end{array}$ & $\begin{array}{c}\Delta \mathrm{Sr}^{\mathrm{b}} \\
(\mathrm{mol})\end{array}$ & $\begin{array}{c}\Sigma \mathrm{Sr}^{2+}{ }_{\text {avail. }}{ }^{\mathrm{c}} \\
\text { (mol) }\end{array}$ & $\begin{array}{c}\text { Recryst. } \\
(\%)\end{array}$ & $\begin{array}{c}\mathrm{Chalk}^{\mathrm{e}} \\
(\%)\end{array}$ \\
\hline 572 & $1.48 \times 10^{2}$ & $0.2 \times 10^{-3}$ & $5.5 \times 10^{-2}$ & 46 & 50 \\
573 & $3.87 \times 10^{2}$ & $0.2 \times 10^{-3}$ & $1.63 \times 10^{-1}$ & 53 & 60 \\
574 & $3.25 \times 10^{2}$ & $0.4 \times 10^{-3}$ & $1.84 \times 10^{-1}$ & 30 & 40 \\
\hline
\end{tabular}

a $\mathrm{\Sigma} \mathrm{CaCO}_{3}=$ moles of calcite in Zones II and III.

${ }^{b} \mathrm{Sr}=$ difference between $\mathrm{Sr} / \mathrm{Ca}$ ratios, mean of observed values and mean of calculated equilibrium in Zone II.

c $\Sigma \mathrm{Sr}^{2}+$ avail. $=$ amount of $\mathrm{Sr}^{2}+$ produced from calcite recrystallization ( $\Sigma$ $\mathrm{Sr}^{2+}$, Table 6) plus amount $\mathrm{Sr}^{2+}$ in solids above calculated equlibrium value: $\Sigma \mathrm{Sr}^{2}+$ avail. $=\left(\Sigma \mathrm{CaCO}_{3} \times \Delta \mathrm{Sr}\right)+\Sigma \mathrm{Sr}^{2}+$

$\mathrm{d}_{\text {Recryst. }}=$ amount of calcite recrystallization: Recryst. $(\%)=$ $\frac{\sum \mathrm{Sr}^{2+}}{\sum \mathrm{Sr}} \times 100$.

$\sum \mathrm{Sr}_{\text {avail. }}$.

${ }^{e}$ Chalk $=$ observed percentages of chalk in sediments from Zones II and III.

served value within Zone II of each site. The chalk estimates represent the thicknesses of all-chalk sections plus one-third of the thicknesses of the intervals of chalk and ooze interlayering, which is an approximation determined from the visual core descriptions.

The highest strontium production rate occurs at Site 572 (Table 7). The carbonate accumulation rate is the highest at this site as well, which supports the contention that high accumulation rates can enhance diagenesis. Overall, the $\mathrm{Sr}^{2+}$ production rates are similar to those reported by Baker et al. (1982) for carbonate-rich DSDP sites drilled in plateau regions in the western Pacific and southern Atlantic. Production rates from 1.8 to $7.6 \times$ $10^{-21} \mathrm{~mol} \mathrm{Sr}^{2+} / \mathrm{cm}^{3} / \mathrm{sec}$ were calculated from downhole changes in $\mathrm{Sr} / \mathrm{Ca}$ ratios of bulk carbonate samples according to their model. These values were recalculated to be expressed in units of moles per cubic centimeter of bulk sediment, as shown in Table 7. These slightly higher production rates may reflect differences in solution susceptibility of calcareous microfossil assemblages. The plateau areas, which lie higher above the lysocline than the sites drilled on Leg 85 , should contain a higher proportion of delicate calcareous microfossils. Consequently, higher reaction rates and greater strontium production may be expected there.

The percentage of recrystallization calculated from the $\mathrm{Sr} / \mathrm{Ca}$ distributions shows very good agreement with the estimated percentage of chalk (Table 8). Detailed petrographic study of the sediments from these sites, coupled with more rigorous and constrained modeling of the $\mathrm{Sr} / \mathrm{Ca}$ distributions, should allow for improved estimates of calcite recrystallization. It is especially important to determine if significant recrystallization occurs at shallower burial depths than is assumed in the model proposed here. Modeling the dissolved $\mathrm{Sr}^{2+}$ profiles will determine if the increases in Zone I are due solely to diffusion from deeper zones.

\section{BIOGENIC SILICA DIAGENESIS}

Centimeter-thick pieces of finely laminated porcelanite were recovered at Site 572. X-ray diffraction analyses indicate that the main silica phase present in these indurated sediments is opal-CT (Jones and Segnit, 1971).
Scanning electron microscopic examination of these samples revealed that the opal-CT occurs predominantly as the massive type (Keene, 1976).

The first sample of porcelanite was found in a core taken at $315 \mathrm{~m}$ corresponding to an age of $12 \mathrm{Ma}$ (see Site 572 chapter). Assuming a $40^{\circ} \mathrm{C} / \mathrm{km}$ geothermal gradient and a $1^{\circ} \mathrm{C}$ bottom-water temperature, the projected temperature at this depth is about $14^{\circ} \mathrm{C}$. The low temperature and young age are at odds with most observed occurrences of opal-CT from other DSDP sites. Kastner (1981) derived two equations relating time and temperature to the opal-A to opal-CT transition by using data from 37 DSDP legs. Applying a temperature of $14^{\circ} \mathrm{C}$, the corresponding age estimates are 50 and $46 \mathrm{Ma}$ ( \pm 5 to \pm 10 ), respectively, for the two equations. The oldest sediment recovered at this site (which was drilled to basement) was less than $16 \mathrm{Ma}$ in age. The carbonate content and sedimentation rates at this site are such that transformation of opal-A to opal-CT should be fostered. However, higher temperatures than those assumed from typical oceanic geothermal gradients would be required to occur or to have occurred in the past. Using the ages of the sediments in the interval where the porcelanites are found (12 to $15 \mathrm{Ma})$, temperature estimates of about 35 and $45^{\circ} \mathrm{C}$ would be needed according to the two equations. Similar temperature estimates are indicated by data compiled from deep-sea siliceous deposits throughout the circum-Pacific area (Hein et al., 1978).

If the higher temperatures implied from the age-temperature relationships of the opal-A to opal-CT transformation actually occurred, they would have greatly enhanced any other diagenetic reactions occurring in these sediments. This is especially important in carbonate recrystallization. The higher temperatures would help explain the high $\mathrm{Sr} / \mathrm{Ca}$ ratios observed in the deeper zones of this site. For example, if a temperature of $40^{\circ} \mathrm{C}$ were applicable to the observed $\mathrm{Sr} / \mathrm{Ca}$ distributions in pore waters, using the temperature-corrected distribution coefficients, the calculated equilibrium values would equal the observed near-constant $\mathrm{Sr} / \mathrm{Ca}$ ratios of $\sim 2.2 \times 10^{-3}$ in the carbonates at Site 572 (Figure 7, Table 3). Whether or not similarly higher temperatures help explain the $\mathrm{Sr}$ / $\mathrm{Ca}$ distributions at the other sites is uncertain. However, it is clear that the possible causes of the high temperatures, and the temporal and spatial significance of the mechanisms and processes causing the higher thermal gradients, deserve further attention and investigation.

\section{SUMMARY AND CONCLUSIONS}

The interstitial water chemistry from sediments recovered during Leg 85 reflects influences from diagenetic reactions common in deep-sea biogenic deposits. Increases in dissolved strontium and silica result from calcite recrystallization and dissolution of amorphous silica, respectively. Minor decreases in sulfate concentrations and increases in alkalinity most likely indicate degradation of buried organic material. Small gradients in calcium and magnesium imply minor or no influence from alteration of volcanic matter or oceanic layer II. The chemical gradients of all species determined are of similar steepness and magnitude; the maxima and min- 
ima, however, occur at different depths. The differences are attributable to variations in diagenetic alterations, sediment composition, and sedimentation rate histories among the sites.

Comparisons between $\mathrm{Sr} / \mathrm{Ca}$ distribution profiles of interstitial waters and bulk carbonate sediments are used to infer locations of calcite recrystallization and allow for calculations of the extent of recrystallization at each site. In the uppermost, nonrecrystallized sediments, variations in dissolved $\mathrm{Sr}^{2+}$ in pore waters are assumed to result from diffusive processes from the underlying zones of active recrystallization. The varying $\mathrm{Sr} / \mathrm{Ca}$ ratios in solids reflect differences in calcareous microfossil content, in diagenetic histories, and possibly in changes in seawater $\mathrm{Sr} / \mathrm{Ca}$ with time. A model estimating the amount of carbonate recrystallization at each site is based on interpretations of dissolved strontium profiles and comparisons between predicted equilibrium and observed $\mathrm{Sr} /$ $\mathrm{Ca}$ ratios in the solids. Accordingly, in zones having experienced recrystallization, between 30 and $53 \%$ of the carbonates have been altered diagenetically. These estimates agree with observed amounts of chalk in the same sediments. At Site 573, more recrystallization is apparent, and it has occurred at shallower depths than at other sites.

Small pieces of laminated porcelanite occur below $300 \mathrm{~m}$ sub-bottom at Site 572. The depth-age relationships of these samples imply significantly higher temperatures than ones inferred from typical oceanic geothermal gradients, possibly by as much as 20 to $30^{\circ} \mathrm{C}$. These higher temperatures may help explain the relatively high $\mathrm{Sr} / \mathrm{Ca}$ in the altered carbonates observed at this site.

\section{ACKNOWLEDGMENTS}

I thank all those responsible for the success of DSDP Leg 85. Special thanks are extended to Mr. Ken Thomson, shipboard chemist, for his excellent work, despite having a tremendous work load during the cruise. Drs. Miriam Kastner and Joris Gieskes, SIO, Paul A. Baker, Duke University, and Ken Johnson, University of California, Santa Barbara, provided stimulating discussions and helpful comments on the topics covered in this report. Financial support for this research came from ONR Contract No. USN N00014-80-C-0440 to M. Kastner.

\section{REFERENCES}

Baker, P. A., Gieskes, J. M., and Elderfield, H., 1982. Diagenesis of carbonates in deep-sea sediments-evidence from $\mathrm{Sr} / \mathrm{Ca}$ ratios and interstitial dissolved $\mathrm{Sr}^{2+}$ data. J. Sediment. Petrol., 52:71-82.

Baker, P. A., Kastner, M., Byerlee, J. D., and Lockner, D. A., 1980. Pressure solution and hydrothermal recrystallization of carbonate sediments-an experimental study. Mar. Geol., 38:185-203.

Bender, M. L., Lorens, R. E., and Williams, D. F., 1975. Sodium, magnesium and strontium in the tests of planktonic foraminifera. Micropaleontology, 21:448-459.

Berner, R. A., 1978. Rate control of mineral dissolution under earth surface conditions. Am. J. Sci., 278:1235-1252. , 1980. Early Diagenesis: A Theoretical Approach: Princeton (Princeton Univ. Press)

Cronblad, H. G., and Malmgren, B. A., 1981. Climatically controlled variation of $\mathrm{Sr}$ and $\mathrm{Mg}$ in Quaternary planktonic foraminifera. Nature (London), 291:61-64.

Elderfield, H., and Gieskes, J. M., 1982. Sr isotopes in interstitial waters of marine sediments from Deep Sea Drilling Project cores. Nature (London), 300:493-497.

Elderfield, H., Gieskes, J. M., Baker, P. A., Oldfield, R. K., Hawkesworth, C. J., and Miller, R., $1982 .{ }^{87} \mathrm{Sr} /{ }^{86} \mathrm{Sr}$ and ${ }^{18} \mathrm{O} /{ }^{16} \mathrm{O}$ ratios, interstitial water chemistry and diagenesis in deep-sea carbonate sediments of the Ontong Java Plateau. Geochim. Cosmochim. Ac$t a, 46: 2259-2268$.

Garrison, R. E., 1981. Diagenesis of oceanic carbonate sediments: A review of the DSDP perspective. In Warme, T. E., Douglas, R. C., and Winterer, E. L. (Eds.), The Deep Sea Drilling Project: A Decade of Progress. Soc. Econ. Paleontol. Mineral. Spec. Publ., 32: 181-207.

Gieskes, J. M., 1973. Interstitial water studies, Leg 15-alkalinity, $\mathrm{pH}, \mathrm{Mg}, \mathrm{Ca}, \mathrm{Si}, \mathrm{PO}_{4}$. In Heezen, B. C., MacGregor, I. D., et al., Init. Repts. DSDP, 20: Washington (U.S. Govt. Printing Office), 813-829.

1974. Interstitial water studies, Leg 25. In Simpson, E. S. W., Schlich, R., et al., Init. Repts. DSDP, 25: Washington (U.S. Govt. Printing Office), 361-394.

1975. Chemistry of interstitial waters of marine sediments. Annu. Rev. Earth Planet. Sci. Lett., 3:433-453.

1978. Reactions controlling strontium abundances in natural waters. In Wedepohl, K. H. (Ed.), Handbook of Geochemistry, II/4, 38-H:1-5.

1981. Deep Sea Drilling interstitial water studies: Implications for chemical alteration of the oceanic crust, layers I and II. In Warme, J. E., Douglas, R. G., and Winterer, E. L. (Eds.), The Deep Sea Drilling Project: A Decade of Progress, Soc. Econ. Paleontol. Mineral. Spec. Publ., 32:149-167.

Gieskes, J. M., Elderfield, H., Lawrence, J. R., Johnson, J., Meyers, B., and Campbell, A., 1982. Geochemistry of interstitial waters and sediments, Leg 64, Gulf of California. In Curray, J. R., Moore, D. G., et al., Init. Repts. DSDP, 64: Washington (U.S. Govt. Printing Office), 675-694.

Gieskes, J. M., and Johnston, K., 1985. Interstitial water studies, Leg 81. In Roberts, D. G., Schnitker, D., et al., Init. Repts. DSDP, 81: Washington (U.S. Govt. Printing Office), 829-836.

Gieskes, J. M., and Lawrence, J. R., 1981. Alteration of volcanic matter in deep-sea sediments: Evidence from the chemical composition of interstitial waters from deep sea drilling cores. Geochim. Cosmochim. Acta, 45:1687-1703.

Graham, D. W., Bender, M. L., Williams, D. F., and Keigwin, Jr., L. D., 1982. Strontium-calcium ratios in Cenozoic planktonic foraminifera. Geochim. Cosmochim. Acta, 46:1281-1292.

Hein, J. R., Scholl, D. W., Barron, J. A., Jones, M. G., and Miller, J., 1978. Diagenesis of late Cenozoic diatomaceous deposits and formation of the bottom simulating reflector in the southern Bering Sea. Sedimentology, 25:155-182.

Holland, H. D., Holland, H. J., and Munoz, J. L., 1964. The coprecipitation of cations with $\mathrm{CaCO}_{3}$-II. The coprecipitation of $\mathrm{Sr}^{2+}$ with calcite between 90 and $100^{\circ} \mathrm{C}$. Geochim. Cosmochim. Acta, 28:1287-1302.

Jones, J. B., and Segnit, E. R., 1971. The nature of opal. I. Nomenclature and constituent phases. J. Geol. Soc. Austr., 18:56-68.

Kastner, M., 1981. Authigenic silicates in deep-sea sediments: Formation and diagenesis. In Emiliani, C. (Ed.), The Sea (Vol. 7), The Oceanic Lithosphere: New York (Wiley).

Katz, A., Sass, E., Starinsky, A., and Holland, H. D., 1972. Strontium behavior in the aragonite-calcite transformation: An experimental study at $40-98^{\circ} \mathrm{C}$. Geochim. Cosmochim. Acta, 36:481-496.

Keene, J. B., 1976. The Distribution, Mineralogy, and Petrography of Biogenic and Authigenic Silica from the Pacific Basin [Ph.D. dissert.]. Scripps Institution of Oceanography, La Jolla.

Kinsman, D. J., 1969. Interpretation of $\mathrm{Sr}^{2+}$ concentration in carbonate minerals and rocks. J. Sediment. Petrol., 39:486-508.

Lawrence, J. R., Gieskes, J. M., and Broecker, W. S., 1975. Oxygen isotope and cation composition of DSDP pore water and the alteration of layer II basalts. Earth Planet. Sci. Lett., 27:1-10.

Lorens, R. B., Bender, M. L., and Williams, D. F., 1977. The early nonstructural chemical diagenesis of foraminiferal calcite. J. Sediment. Petrol., 47:1602-1609.

McDuff, R. E., and Gieskes, J. M., 1976. Calcium and magnesium profiles in DSDP interstitial waters: Diffusion or reaction? Earth Planet. Sci. Lett., 33:1-10.

Manheim, F. T., and Waterman, L. S., 1974. Diffusimetry (diffusion constant estimation) on sediment cores by resistivity probe. In von der Borch, C. C., Sclater, J. G., et al., Init. Repts. DSDP, 22: Washington (U.S. Govt. Printing Office), 663-670. 
Matter, A., Douglas, R. G., and Perch-Nielsen, K., 1975. Fossil preservation, geochemistry and diagenesis of carbonates from the Shatsky Rise, N.W. Pacific. In Larson, R. L., Moberly, R., et al., Init. Repts. DSDP, 32: Washington (U.S. Govt. Printing Office), 891-921.

Mucci, A., and Morse, J. W., 1983. The incorporation of $\mathrm{Mg}^{2+}$ and $\mathrm{Sr}^{2+}$ into calcite overgrowths: Influences of growth rate and solution composition. Geochim. Cosmochim. Acta., 47:217-233.

Sayles, F. L., and Manheim, F. T., 1975. Interstitial solutions and diagenesis in deeply buried marine sediments: Results from the Deep Sea Drilling Project. Geochim. Cosmochim. Acta, 39:103-127.

Schlanger, S. O., and Douglas, R. G., 1974. The pelagic ooze-chalklimestone transition and its implications for marine stratigraphy. In Hsü, K. J., and Jenkyns, H. C. (Eds.), Pelagic Sediments: On
Land and Under the Sea. Internat. Assoc. Sedimentologists Spec. Publ., 1:117-148.

van der Lingen, G. J., and Packham, G. H., 1975. Relationships between diagenesis and physical properties of biogenic sediments of the Ontong-Java Plateau (Sites 288 and 289, Deep Sea Drilling Project). In Andrews, J. E., Packham, G., et al., Init. Repts. DSDP, 30: Washington (U.S. Govt. Printing Office), 443-481.

Von Herzen, R. P., Fiske, R. J., and Sutton, G. H., 1971. Geothermal gradients on Leg 8. In Tracey, J. I., Jr., Sutton, G. H., et al., Init. Repts. DSDP, 8: Washington (U.S. Govt. Printing Office), 837-849.

Date of Initial Receipt: 14 September 1983

Date of Acceptance: 29 March 1984 